Supporting Information for

\title{
Rate constants for Anilidyl Radical Cyclization Reactions
}

\author{
E. Martinez II, M. Newcomb* \\ Department of Chemistry, University of Illinois at Chicago, 845 W. Taylor St., Chicago, IL 60607
}

Table of Contents

Table of Contents

Supplemental Experimental Section

References for Supporting Information

NMR Spectra
S1

S2-S5

S5

S6-S45 


\section{Supplemental Experimental Section}

5, 5-Diphenyl-4-pentenoic acid (A) and 6, 6-diphenyl-5-hexenoic acid (B) were prepared as previously described. ${ }^{\mathrm{S} 1, \mathrm{~S} 2}$

Preparation of anilides 1. In a flame-dried, nitrogen-flushed round bottomed flask equipped with a stirbar and bubbler was dissolved $1.0 \mathrm{mmol}$ of the respective acid and 3 drops of DMF in $10 \mathrm{~mL}$ of $\mathrm{CH}_{2} \mathrm{Cl}_{2}$. 1.1 equiv of oxalyl chloride was added dropwise, and the solution was stirred until bubbling subsided (ca. $30 \mathrm{~min}$ ). The mixture was concentrated in vacuo, the residue was redissolved in $10 \mathrm{~mL}$ of $\mathrm{CH}_{2} \mathrm{Cl}_{2}$, and the mixture was again concentrated in vacuo. The acid chloride was dissolved in $10 \mathrm{~mL}$ of $\mathrm{CH}_{2} \mathrm{Cl}_{2}$ and a solution of 1.05 equiv of the respective aniline and 1.05 equiv of $\mathrm{Et}_{3} \mathrm{~N}$ in $5 \mathrm{~mL}$ of $\mathrm{CH}_{2} \mathrm{Cl}_{2}$ was added. The mixture was stirred for $30 \mathrm{~min}$, diluted with $50 \mathrm{~mL}$ of $\mathrm{CH}_{2} \mathrm{Cl}_{2}$, and washed with $3 \times 40 \mathrm{~mL} 0.1 \mathrm{M} \mathrm{HCl}$ solution, $2 \times$ $20 \mathrm{~mL}$ of water and $20 \mathrm{~mL}$ brine, and dried over $\mathrm{MgSO}_{4}$. Concentration of the organic layer yielded oily crystals, which were dissolved in a polar solvent with warming. The anilides were precipitated by slow addition of petroleum ether, hexanes or cyclohexane with cooling at $0{ }^{\circ} \mathrm{C}$. A second precipitation yielded anilides of high purity, which were then dried under vacuum.

Sulfenamide Preparation. Method B, described in the text of the paper, was used for the preparation of $\mathbf{2 b - 2 f}$. Selfenamide $\mathbf{2 a}$ was prepared by the following procedure (Method A). In a flame-dried, round-bottomed flask equipped with a stirbar under static nitrogen was dissolved $1.0 \mathrm{mmol}$ of the anilide $1 \mathrm{a}$ in $10 \mathrm{~mL}$ of THF at room temperature. To this solution was added via syringe, 1.05 equivalents of $1.6 \mathrm{M} \mathrm{n}$-BuLi in hexanes over $5 \mathrm{~min}$. The flask was cooled to $-78^{\circ} \mathrm{C}$, and the remainder of the reaction, work-up, and purification was carried out as described in Method B.

Preparation of lactams 5 are described in the text of the paper.

$N$-Phenyl-5,5-diphenyl-4-pentenamide (1a) was obtained in $84 \%$ yield from $17.8 \mathrm{mmol}$ of acid $\mathbf{A}$. The crude crystals were twice recrystallized from $\mathrm{CH}_{2} \mathrm{Cl}_{2}-$ cyclohexane $(1: 1, \mathrm{v}: \mathrm{v})$ to yield off-white, sheet-like crystals. Mp 135-138 ${ }^{\circ} \mathrm{C} .{ }^{1} \mathrm{H} \mathrm{NMR}\left(\mathrm{CDCl}_{3}\right): \delta$ 7.47-7.04 (m, $\left.15 \mathrm{H}\right)$, $6.09(\mathrm{t}, J=7.2 \mathrm{~Hz}, 1 \mathrm{H}), 2.56-2.40(\mathrm{~m}, 4 \mathrm{H})\left(\mathrm{NH}\right.$ not observed). ${ }^{13} \mathrm{C}$ NMR: $\delta 170.5,143.2$, $142.2,139.6,137.8,129.7,128.9,128.3,128.1,127.2$, 127.1, 124.2, 119.8, 37.6, 25.8. HRMS $(\mathrm{M}+\mathrm{H})^{+}$: calcd for $\mathrm{C}_{23} \mathrm{H}_{22} \mathrm{NO}, 328.1701$; found 328.1696 .

$N$-(4-Ethylphenyl)-5,5-diphenyl-4-pentenamide (1b) was obtained in 78\% yield from $29.8 \mathrm{mmol}$ of acid $\mathbf{A}$. The amide was twice precipitated from $\mathrm{CH}_{2} \mathrm{Cl}_{2}$ with hexanes to give the amide as finely-divided, white needles. $\mathrm{Mp} 138-140{ }^{\circ} \mathrm{C} .{ }^{1} \mathrm{H} \mathrm{NMR}\left(\mathrm{CDCl}_{3}\right): \delta 7.44-7.07(\mathrm{~m}, 14$ $\mathrm{H}), 6.08(\mathrm{t}, J=7.1 \mathrm{~Hz}, 1 \mathrm{H}), 2.58-2.41(\mathrm{~m}, 6 \mathrm{H}), 1.19(\mathrm{t}, J=7.5 \mathrm{~Hz}, 3 \mathrm{H})(\mathrm{NH}$ not observed). ${ }^{13}$ C NMR: $\delta 170.4,143.1,142.2,140.2,139.6,135.4,129.7,128.3,128.2,128.1,127.3,127.2$, 127.1, 127.1, 120.0, 37.5, 28.2, 25.8, 15.6. HRMS $(\mathrm{M}+\mathrm{H})^{+}$: calcd for $\mathrm{C}_{25} \mathrm{H}_{26} \mathrm{NO}, 356.2014$; found 356.2011 .

$\mathrm{N}$-(4-Fluorophenyl)- 5,5-diphenyl-4-pentenamide (1c) was obtained in 57\% yield from $7.93 \mathrm{mmol}$ of acid $\mathbf{A}$. The amide was twice precipitated from $\mathrm{CH}_{2} \mathrm{Cl}_{2}$ with hexanes to give the amide as off-white, fine needles. Mp 120-122 ${ }^{\circ} \mathrm{C}$. ${ }^{1} \mathrm{H} \mathrm{NMR}\left(\mathrm{CDCl}_{3}\right): \delta 7.57(\mathrm{br} \mathrm{s}, 1 \mathrm{H}), 7.40-$ $7.14(\mathrm{~m}, 12 \mathrm{H}), 6.93(\mathrm{t}, J=8.4 \mathrm{~Hz}, 2 \mathrm{H}), 6.06(\mathrm{t}, J=6.9 \mathrm{~Hz}, 1 \mathrm{H}), 2.54-2.40(\mathrm{~m}, 4 \mathrm{H}) .{ }^{13} \mathrm{C}$ NMR: $\delta 170.6,161.8,160.9,157.6,143.2,142.2,139.6,133.8$. 129.7, 128.3, 128.1, 127.2, 127.1, 121.8, 121.7, 101.1, 37.4, 25.8. HRMS (M+H) ${ }^{+}$: calcd for $\mathrm{C}_{23} \mathrm{H}_{21} \mathrm{FNO}, 346.1607$; found 346.1606 .

$\mathrm{N}$-(4-Acetylphenyl)-5,5-diphenyl-4-pentenamide (1d) was obtained in 89\% yield from $9.0 \mathrm{mmol}$ of acid $\mathbf{A}$. The crude reaction mixture was eluted through a short column of silica gel 
(ca. $50 \mathrm{~g}$ ) with 1:3 ethyl acetate/hexanes, and the crude amide was twice recrystallized from 1:1 $\mathrm{CH}_{2} \mathrm{Cl}_{2}$ - cyclohexane to yield pale yellow, finely divided needles. Mp 122-124 ${ }^{\circ} \mathrm{C}$. ${ }^{1} \mathrm{H} \mathrm{NMR}$ $\left(\mathrm{CDCl}_{3}\right): \delta 7.91-7.13(\mathrm{~m}, 14 \mathrm{H}), 6.08(\mathrm{t}, J=6.8 \mathrm{~Hz}, 1 \mathrm{H}), 2.54-2.50(\mathrm{~m}, 7 \mathrm{H})(\mathrm{NH}$ not observed). ${ }^{13}$ C NMR: $\delta$ 197.2, 143.4, 142.4, 142.1, 139.5, 132.6, 129.7, 128.3, 128.1, 127.2, 126.9, 118.9, 37.7, 26.4, 25.6. HRMS $(\mathrm{M}+\mathrm{H})^{+}$: calcd for $\mathrm{C}_{25} \mathrm{H}_{24} \mathrm{NO}_{2}$, 370.1807; found 370.1826.

$\mathrm{N}$-(4-Cyanophenyl)-5,5-diphenyl-4-pentenamide (1e) was obtained in 38\% yield from $10.3 \mathrm{mmol}$ of acid $\mathbf{A}$. The crude reaction mixture was purified by chromatography on silica gel with 1:1 ethyl acetate - hexanes elution, and the amide was further purified by precipitation from $\mathrm{CH}_{2} \mathrm{Cl}_{2}$ with hexanes to give white, finely divided needles. $\mathrm{Mp} 150-152{ }^{\circ} \mathrm{C}$. ${ }^{1} \mathrm{H} \mathrm{NMR}\left(\mathrm{CDCl}_{3}\right)$ : $\delta 7.88(\mathrm{~s}, 1 \mathrm{H}), 7.65-7.13(\mathrm{~m}, 13 \mathrm{H}), 6.07(\mathrm{t}, J=6.9 \mathrm{~Hz}, 1 \mathrm{H}), 2.59-2.46(\mathrm{~m}, 4 \mathrm{H})(\mathrm{NH}$ not observed). ${ }^{13} \mathrm{C}$ NMR: $\delta 171.1,143.5,142.1,142.0,139.5,133.2,129.6,128.3,128.1,127.3$, 127.2, 126.7, 119.4, 118.9, 106.6, 37.6, 29.5. HRMS $(\mathrm{M}+\mathrm{H})^{+}$: calcd for $\mathrm{C}_{24} \mathrm{H}_{21} \mathrm{~N}_{2} \mathrm{O}, 353.1654$; found 353.1658 .

$\mathrm{N}$-(4-Methoxyphenyl)-5,5-diphenyl-4-pentenamide (1f) was obtained in 55\% yield from $7.91 \mathrm{mmol}$ of acid A. Precipitation from $\mathrm{CH}_{2} \mathrm{Cl}_{2}$ with hexanes produced white, finelydivided needles. Mp147-148 ${ }^{\circ} \mathrm{C} .{ }^{1} \mathrm{H}$ NMR $\left(\mathrm{CDCl}_{3}\right): \delta 7.36-7.16(\mathrm{~m}, 13 \mathrm{H}), 6.81(\mathrm{~d}, J=9.3 \mathrm{~Hz}$, $2 \mathrm{H}), 6.09(\mathrm{t}, J=7.5 \mathrm{~Hz}, 1 \mathrm{H}), 3.75(\mathrm{~s}, 3 \mathrm{H}), 2.55-2.41(\mathrm{~m}, 4 \mathrm{H}) .{ }^{3} \mathrm{C}$ NMR: $\delta 170.3,161.8$, 156.3, 143.1, 142.3, 139.7, 130.9, 129.8, 128.3, 128.1, 127.4, 127.2, 127.1, 121.8, 114.1, 55.4, 37.4, 25.9. HRMS $(\mathrm{M}+\mathrm{H})^{+}$: calcd for $\mathrm{C}_{24} \mathrm{H}_{24} \mathrm{NO}_{2}, 358.1807$; found 358.1813 .

$N$-Phenyl- $N$-(thiophenyl)-5,5-diphenyl-4-pentenamide (2a) was obtained in $80 \%$ yield from $5.14 \mathrm{mmol}$ of 1a. Column chromatography on silica gel using 1:9 ethyl acetate--hexanes afforded $2 \mathrm{a}$ as a tan, viscous oil. ${ }^{1} \mathrm{H}$ NMR $\left(\mathrm{CDCl}_{3}\right): \delta 7.31-7.10(\mathrm{~m}, 20 \mathrm{H}), 6.09(\mathrm{t}, J=7.5 \mathrm{~Hz}, 1$ $\mathrm{H}), 2.67$ (br s, $\left.2 \mathrm{H}, \alpha-\mathrm{CH}_{2}\right), 2.52-2.45(\mathrm{~m}, 2 \mathrm{H}) .{ }^{13} \mathrm{C}$ NMR: $\delta 175.5,144.6,142.6,142.1,139.5$, $137.3,129.6,129.1,128.1,127.9,127.4,127.0,126.9,126.7,126.2,34.4,25.7$. HRMS [M+H] $]^{+}$: calcd for $\mathrm{C}_{29} \mathrm{H}_{26} \mathrm{NOS}, 436.1735$; found 436.1742 .

$\boldsymbol{N}$-(4-Ethylphenyl)- $\boldsymbol{N}$-(thiophenyl)-5,5-diphenyl-4-pentenamide (2b) was obtained in $49 \%$ yield from 16.9 mmol of $\mathbf{1 b}$. Column chromatography on silica gel using $\mathrm{CH}_{2} \mathrm{Cl}_{2}$ yielded sulfenamide $\mathbf{2 b}$ as a pale yellow-green oil. ${ }^{1} \mathrm{H}$ NMR $\left(\mathrm{CDCl}_{3}\right): \delta 7.32-7.00(\mathrm{~m}, 19 \mathrm{H}), 6.08(\mathrm{t}, J=$ $7.2 \mathrm{~Hz}, 3 \mathrm{H}), 2.61-2.44(\mathrm{~m}, 6 \mathrm{H}), 1.17(\mathrm{t}, J=7.5 \mathrm{~Hz}, 3 \mathrm{H}) .{ }^{13} \mathrm{C}$ NMR: $\delta 175.5,143.5,142.5$, $142.3,142.2$, 139.5, 137.5, 130.2, 129.6, 129.0, 128.5, 128.1, 127.9, 127.5, 127.2, 127.0, 126.8, 126.5, 126.1, 34.3, 28.2, 25.7, 15.1. HRMS $(\mathrm{M}+\mathrm{H})^{+}$: calcd for $\mathrm{C}_{31} \mathrm{H}_{30} \mathrm{NOS}, 464.2048$; found 464.2036.

$N$-(4-Fluorophenyl)- $N$-(thiophenyl)-5,5-diphenyl-4-pentenamide (2c) was obtained in $63 \%$ yield from 1.14 mmol of 1c. Column chromatography on silica gel using 1:15 ethyl acetate/hexanes yielded sulfenamide $2 \mathrm{c}$ as a yellow oil. ${ }^{\mathrm{H}} \mathrm{H} \mathrm{NMR}\left(\mathrm{CDCl}_{3}\right): \delta$ 7.35-6.90 (m, 17 $\mathrm{H}), 6.92(\mathrm{t}, J=16.5 \mathrm{~Hz}, 2 \mathrm{H}), 6.10(\mathrm{t}, J=7.5 \mathrm{~Hz}, 1 \mathrm{H}), 2.71\left(\right.$ br. $\left.\mathrm{s}, 2 \mathrm{H}, \alpha-\mathrm{CH}_{2}\right), 2.53-2.45(\mathrm{~m}, 2$ H). ${ }^{13} \mathrm{C}$ NMR: $\delta 175.7,162.9,159.6,142.8,142.2,140.6,139.6,137.1,129.6,129.2,128.5$, $128.4,128.1,128.0,127.7,127.3,127.1,126.9,116.0,115.7,34.3,25.7$. HRMS $(\mathrm{M}+\mathrm{H})^{+}$: calcd for $\mathrm{C}_{29} \mathrm{H}_{25} \mathrm{NOFS}, 454.1641$; found 454.1666.

$N$-(4-Acetylphenyl)- $N$-(thiophenyl)-5,5-diphenyl-4-pentenamide (2d) was obtained in $15 \%$ yield from $0.47 \mathrm{mmol}$ of $\mathbf{1 d}$. Column chromatography on silica gel using 1:30 ethyl acetate/methylene chloride yielded sulfenamide $2 \mathbf{d}$ as a yellow oil. ${ }^{1} \mathrm{H} \mathrm{NMR}\left(\mathrm{CDCl}_{3}\right): \delta 7.91$ $7.11(\mathrm{~m}, 19 \mathrm{H}), 6.10(\mathrm{t}, J=7.4 \mathrm{~Hz}, 1 \mathrm{H}), 2.82(\mathrm{t}, J=7.6 \mathrm{~Hz}, 2 \mathrm{H}), 2.57-2.47(\mathrm{~m}, 5 \mathrm{H}) .{ }^{13} \mathrm{C} \mathrm{NMR}$ : $\delta 196.7,175.7,148.4,142.9,142.1,139.5,136.9,135.5,130.3,129.6,129.3,129.2,128.2,127.7$, $127.2,127.0,126.7,125.8,122.6,114.0,34.5,26.6,25.7$. HRMS $(\mathrm{M}+\mathrm{H})^{+}$: calcd for $\mathrm{C}_{31} \mathrm{H}_{28} \mathrm{NO}_{2} \mathrm{~S}, 478.1841$; found 478.1860. 
$N$-(4-Cyanophenyl)- $N$-(thiophenyl)- 5,5-diphenyl-4-pentenamide (2e) was obtained in $22 \%$ yield from $3.43 \mathrm{mmol}$ of 1e. Column chromatography on silica gel using 1:6 ethyl acetate/hexanes yielded sulfenamide $2 \mathrm{e}$ as a yellow oil. ${ }^{1} \mathrm{H} \mathrm{NMR}\left(\mathrm{CDCl}_{3}\right): \delta 7.51-7.10(\mathrm{~m}, 19$ $\mathrm{H}), 6.10(\mathrm{t}, J=7.5 \mathrm{~Hz}, 1 \mathrm{H}), 2.87(\mathrm{br} \mathrm{t}, J=6.9 \mathrm{~Hz}, 2 \mathrm{H}), 2.51$ (br q, $J=7.0 \mathrm{~Hz}, 2 \mathrm{H}) .{ }^{13} \mathrm{C} \mathrm{NMR}$ : $\delta 175.7,147.9,142.8,141.9,139.4,136.5,132.6,129.4,129.3,128.1,127.9,127.6,127.1,126.9$, 125.0, 118.0, 110.2, 34.1, 25.4. . HRMS $(\mathrm{M}+\mathrm{H})^{+}$: calcd for $\mathrm{C}_{30} \mathrm{H}_{25} \mathrm{~N}_{2} \mathrm{OS}, 461.1688$; found 461.1695 .

$\mathrm{N}$-(4-Methoxyphenyl)- $\mathrm{N}$-(thiophenyl)-5,5-diphenyl-4-pentenamide (2f) was obtained in $68 \%$ yield from $1.12 \mathrm{mmol}$ of 1f. Column chromatography on silica gel using 1:4 ethyl acetate/hexanes yielded sulfenamide $2 \mathrm{f}$ as a yellow-green oil. ${ }^{1} \mathrm{H}$ NMR $\left(\mathrm{CDCl}_{3}\right): \delta 7.36-6.76(\mathrm{~m}$, $20 \mathrm{H}), 6.08(\mathrm{br} \mathrm{t}, J=6.5 \mathrm{~Hz}, 1 \mathrm{H}), 3.73(\mathrm{~s}, 3 \mathrm{H}), 2.57-2.46(\mathrm{~m}, 3 \mathrm{H}) .{ }^{13} \mathrm{C}$ NMR: $\delta 158.6,142.6$, 142.2, 139.6, 137.7, 137.4, 129.7, 129.1, 128.1, 128.0, 127.6, 127.5, 127.1, 126.9, 114.3, 55.3, 34.5 (broad), 25.8 (carbonyl carbon not observed). HRMS $(\mathrm{M}+\mathrm{H})^{+}$: calcd for $\mathrm{C}_{30} \mathrm{H}_{28} \mathrm{NO}_{2} \mathrm{~S}$, 466.1841; found 466.1821 .

$\mathrm{N}$-Phenyl-5-(diphenylmethyl)-2-pyrrolidinone (5a) was obtained in 8.3\% yield from $3.07 \mathrm{mmol}$ of 2a. Column chromatography on silica gel using 1:3 ethyl acetate/hexanes yielded a clear, colorless oil which solidified upon standing. Recrystallization from 1:10 $\mathrm{CH}_{2} \mathrm{Cl}_{2--}$ hexanes afforded lactam 5a as clear, colorless prisms. Mp 128-131 ${ }^{\circ} \mathrm{C}$. ${ }^{1} \mathrm{H}$ NMR $\left(\mathrm{d}_{6}\right.$-acetone): $\delta$ 7.60-7.09 (m, $15 \mathrm{H}), 5.38-5.34(\mathrm{~m}, 1 \mathrm{H}), 4.40(\mathrm{~d}, J=4.2 \mathrm{~Hz}, 1 \mathrm{H}), 2.54-2.40(\mathrm{~m}, 1 \mathrm{H}), 2.19-2.01$ $(\mathrm{m}, 2 \mathrm{H}), 1.51(\mathrm{dt}, J=16.5 \mathrm{~Hz}, 10.2 \mathrm{~Hz}, 1 \mathrm{H}) .{ }^{13} \mathrm{C}$ NMR: $\delta 173.9,142.3,141.5,139.4,130.0$, 129.3, 129.1, 129.0, 127.5, 127.2, 125.3, 123.9, 61.8, 52.5, 31.3, 22.0. HRMS (M+Na) $)^{+}$: calcd for $\mathrm{C}_{23} \mathrm{H}_{21} \mathrm{NONa}, 350.1521$; found 350.1524 .

$\mathrm{N}$-(4-Ethylphenyl)-5-(diphenylmethyl)-2-pyrrolidinone (5b) was obtained in 13\% yield from $2.0 \mathrm{mmol}$ of $\mathbf{2 b}$. Column chromatography on silica gel using 1:4 ethyl acetate--exanes yielded a clear, colorless oil which solidified upon standing. Recrystallization from $1: 1 \mathrm{CH}_{2} \mathrm{Cl}_{2^{--}}$ cyclohexane afforded lactam $\mathbf{5 b}$ as clear, colorless prisms. Mp 108-110 ${ }^{\circ} \mathrm{C}$. ${ }^{1} \mathrm{H}$ NMR (acetone$\left.d_{6}\right): \delta 7.50-7.07(\mathrm{~m}, 14 \mathrm{H}), 5.31-5.26(\mathrm{ddd}, J=8.7 \mathrm{~Hz}, 3.6 \mathrm{~Hz}, 1.8 \mathrm{~Hz}, 1 \mathrm{H}), 4.40(\mathrm{~d}, J=3.6 \mathrm{~Hz}$, $1 \mathrm{H}), 2.59(\mathrm{q}, J=7.5 \mathrm{~Hz}, 2 \mathrm{H}), 2.49-2.35(\mathrm{~m}, 1 \mathrm{H}), 2.15-1.97(\mathrm{~m}, 2 \mathrm{H}), 1.51-1.39(\mathrm{~m}, 1 \mathrm{H}), 1.18$ $(\mathrm{t}, J=7.7 \mathrm{~Hz}, 3 \mathrm{H}) .{ }^{13} \mathrm{C}$ NMR: $\delta 173.7,142.2,141.5,141.1,136.9,130.0,129.3,129.0,128.9$, 128.6, 127.4, 127.1, 123.8, 61.8, 52.2, 31.3, 21.9, 16.0. HRMS $(\mathrm{M}+\mathrm{H})^{+}$: calcd for $\mathrm{C}_{25} \mathrm{H}_{26} \mathrm{NO}$, 356.2014 ; found 356.2011 .

$\mathrm{N}$-(4-Fluorophenyl)-5-(diphenylmethyl)-2-pyrrolidinone (5c) was obtained in 46\% yield from $0.61 \mathrm{mmol}$ of 2c. Crystals, which precipitated overnight from the crude reaction mixture, were recrystallized twice from 1:1 $\mathrm{CH}_{2} \mathrm{Cl}_{2}$--hexanes to afford lactam $\mathbf{5 c}$ as clear, colorless prisms. Mp $149-151^{\circ} \mathrm{C}$. ${ }^{1} \mathrm{H}$ NMR (acetone- $\left.d_{6}\right): \delta 7.53-6.99(\mathrm{~m}, 14 \mathrm{H}), 5.35(\mathrm{ddd}, J=$ 8.6 Hz, 5.4 Hz, $1.8 \mathrm{~Hz}, 1 \mathrm{H}), 4.37$ (d, $J=5.1 \mathrm{~Hz}, 1 \mathrm{H}), 2.52-2.38(\mathrm{~m}, 1 \mathrm{H}), 2.17-2.03(\mathrm{~m}, 2 \mathrm{H})$, 1.83-1.69 (m, $1 \mathrm{H}) .{ }^{13} \mathrm{C}$ NMR: $\delta 174.0,161.7,142.3,141.9,139.7,129.7,129.3,129.0,129.0$, $127.5,127.1,126.5,126.3,115.8,115.5,62.5,53.5,31.0,22.7$. HRMS $[\mathrm{M}+\mathrm{H}]^{+}:$calcd for $\mathrm{C}_{23} \mathrm{H}_{21} \mathrm{FNO}, 346.1607$; found 346.1605.

$\mathrm{N}$ - (4-Acetylphenyl)-5-(diphenylmethyl)-2-pyrrolidinone (5d) was obtained in 34\% yield from $0.22 \mathrm{mmol}$ of $\mathbf{2 d}$. Column chromatography on silica gel using 1:3 ethyl acetate-hexanes yielded a pale yellow oil. ${ }^{1} \mathrm{H}$ NMR (acetone- $\left.d_{6}\right): \delta 7.97-7.10(\mathrm{~m}, 14 \mathrm{H}), 5.48(\mathrm{ddd}, J=$ $8.85 \mathrm{~Hz}, 4.65 \mathrm{~Hz}, 1.2 \mathrm{~Hz}, 1 \mathrm{H}), 4.45$ (d, $J=4.8 \mathrm{~Hz}, 1 \mathrm{H}), 2.55$ (s, $3 \mathrm{H}), 2.23-2.04$ (m, $3 \mathrm{H}), 1.65-$ $1.53(\mathrm{~m}, 1 \mathrm{H}) .{ }^{13} \mathrm{C}$ NMR: $\delta 196.6,174.5,143.6,142.0,141.4,133.7,130.0,129.7,129.5,129.1$, 127.7, 127.3, 122.7, 61.7, 52.7, 31.4, 26.5, 22.1. HRMS $(\mathrm{M}+\mathrm{H})^{+}$: calcd for $\mathrm{C}_{25} \mathrm{H}_{24} \mathrm{NO}_{2}$, 370.1807 ; found 370.1802 . 
$N$ - (4-Cyanophenyl)-5-(diphenylmethyl)-2-pyrrolidinone (5e) was obtained in 34\% yield from $0.21 \mathrm{mmol}$ of 2e. Column chromatography on silica gel using 1:3 ethyl acetate-hexanes yielded a clear, colorless oil, which solidified upon standing. Recrystallization from 1:1 $\mathrm{CH}_{2} \mathrm{Cl}_{2}$--cyclohexane afforded the lactam as clear, colorless prisms. Mp 175-177 ${ }^{\circ} \mathrm{C}$. ${ }^{1} \mathrm{H} \mathrm{NMR}$ (acetone- $\left.d_{6}\right): \delta 7.79-7.11(\mathrm{~m}, 14 \mathrm{H}), 5.52(\mathrm{ddd}, J=8.4 \mathrm{~Hz}, 5.55 \mathrm{~Hz}, 1.5 \mathrm{~Hz}, 1 \mathrm{H}), 4.44(\mathrm{~d}, J=5.4$ $\mathrm{Hz}, 1 \mathrm{H}), 2.58-2.44(\mathrm{~m}, 1 \mathrm{H}), 2.23-2.04(\mathrm{~m}, 2 \mathrm{H}), 1.87-1.75(\mathrm{~m}, 1 \mathrm{H}) .{ }^{13} \mathrm{C}$ NMR: $\delta 174.7,143.6$, 141.9, 141.6, 133.2, 129.8, 129.6, 129.2, 129.0, 127.7, 127.3, 123.7, 119.2, 107.4, 61.9, 53.4, 31.3, 22.5. HRMS $(\mathrm{M}+\mathrm{H})^{+}$: calcd for $\mathrm{C}_{24} \mathrm{H}_{21} \mathrm{~N}_{2} \mathrm{O}, 353.1654$; found 353.1646.

$\mathrm{N}$-(4-Methoxyphenyl)-5-(diphenylmethyl)-2-pyrrolidinone (5f) was obtained in 82\% yield from $1.13 \mathrm{mmol}$ of $\mathbf{2 f}$. Column chromatography on silica gel using 1:3 ethyl acetate-hexanes yielded a clear, colorless oil, which solidified upon standing. Recrystallization from 1:4 $\mathrm{CH}_{2} \mathrm{Cl}_{2}$--hexanes afforded lactam $\mathbf{5 f}$ as clear, colorless prisms. Mp 100-102 ${ }^{\circ} \mathrm{C}$. ${ }^{1} \mathrm{H} \mathrm{NMR}$ (acetone- $\left.d_{6}\right): \delta 7.48-6.84(\mathrm{~m}, 14 \mathrm{H}), 5.22-5.19(\mathrm{~m}, 1 \mathrm{H}), 4.35(\mathrm{~d}, J=4.2 \mathrm{~Hz}, 1 \mathrm{H}), 3.71(\mathrm{~s}, 3 \mathrm{H})$, 2.42-2.28 (m, $1 \mathrm{H}), 2.10-1.96(\mathrm{~m}, 2 \mathrm{H}), 1.48(\mathrm{dt}, J=16.8 \mathrm{~Hz}, 10.2 \mathrm{~Hz}, 1 \mathrm{H}) .{ }^{13} \mathrm{C} \mathrm{NMR}: \delta 173.6$, $157.3,142.3,141.4,132.1,129.9,129.2,129.0,128.8,127.4,127.0,125.5,114.4,62.1,55.5$, 52.3, 31.1, 21.9. HRMS $(\mathrm{M}+\mathrm{H})^{+}$: calcd for $\mathrm{C}_{24} \mathrm{H}_{24} \mathrm{NO}_{2}$, 358.1807; found 358.1799.

$N$-(4-Methoxyphenyl)-6,6-diphenyl-5-hexenamide was obtained in 29\% yield from $19.7 \mathrm{mmol}$ of acid $\mathbf{B}$. The amide was twice precipitated from THF with hexanes giving white, finely divided needles. Mp 146-147 ${ }^{\circ} \mathrm{C}$; ${ }^{1} \mathrm{H} \mathrm{NMR}\left(\mathrm{CDCl}_{3}\right)$ : 7.33-714 (m, $\left.15 \mathrm{H}\right), 6.95-6.85$ (m,2 $\mathrm{H}), 6.05(\mathrm{t}, J=7.4 \mathrm{~Hz}, 1 \mathrm{H}), 2.28-2.14(\mathrm{~m}, 4 \mathrm{H}), 1.84$ (quintet, $J=7.5 \mathrm{~Hz}, 2 \mathrm{H})(\mathrm{NH}$ not observed). ${ }^{13} \mathrm{C}$ NMR: $\delta 156.2,142.4,139.9,130.9,129.8,128.7,128.2,128.0,127.1,126.9$, 121.9, 113.9, 55.4, 36.8, 29.1, 25.8. HRMS $(\mathrm{M}+\mathrm{H})^{+}$: calcd for $\mathrm{C}_{25} \mathrm{H}_{26} \mathrm{NO}_{2}, 372.1964$; found 372.1975 .

$N$-(4-Methoxyphenyl)- $N$-(thiophenyl)-6,6-diphenyl-5-hexenamide was obtained in $95 \%$ yield from $3.9 \mathrm{mmol}$ of the above amide. Column chromatography on silica gel using $\mathrm{CH}_{2} \mathrm{Cl}_{2}$ yielded a yellow oil which solidified upon standing. The crystals were recrystallized from 1:3 $\mathrm{CH}_{2} \mathrm{Cl}_{2}$--cyclohexane to give clear crystals. Mp 68-71 ${ }^{\circ} \mathrm{C} .{ }^{1} \mathrm{H} \mathrm{NMR}\left(\mathrm{CDCl}_{3}\right): \delta 7.30$ $6.74(\mathrm{~m}, 19 \mathrm{H}), 5.99(\mathrm{t}, J=7.1 \mathrm{~Hz}, 1 \mathrm{H}), 3.66(\mathrm{~s}, 3 \mathrm{H}), 2.46$ (br. s, $\left.2 \mathrm{H}, \alpha-\mathrm{CH}_{2}\right), 2.12-2.07$ (m, 2 $\mathrm{H}), 1.82$ (qunitet, $J=7.5 \mathrm{~Hz}, 2 \mathrm{H}) .{ }^{13} \mathrm{C}$ NMR: $\delta$ 176.0, 158.4, 142.2, 142.1, 139.7, 137.6, 137.4, 129.5, 128.9, 128.6, 127.9, 127.8, 127.3, 126.9, 126.6, 114.1, 55.1, 33.7 (broad), 29.3, 28.9. HRMS $(\mathrm{M}+\mathrm{H})^{+}$: calcd for $\mathrm{C}_{31} \mathrm{H}_{30} \mathrm{NO}_{2} \mathrm{~S}, 480.1997$; found 480.1993 .

\section{References for Supporting Information}

(S1) Horner, J. H.; Musa, O. M.; Bouvier, A.; Newcomb, M. J. Am. Chem. Soc. 1998, 120, 7738-7748.

(S2) Guthrie, R. W.; Kaplan, G. L.; Mennona, F. A.; Tilley, J. W.; Kierstead, R. W.; Mullin, J. G.; LeMahieu, R. A.; Zawoiski, S.; O'Donnell, M.; Crowley, H.; Yaremko, B.; Welton, A. F. J. Med. Chem. 1989, 32, 1820-1835. 


\section{Compound 1a}<smiles>O=C(CCC=C(c1ccccc1)c1ccccc1)Nc1ccccc1</smiles>

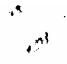
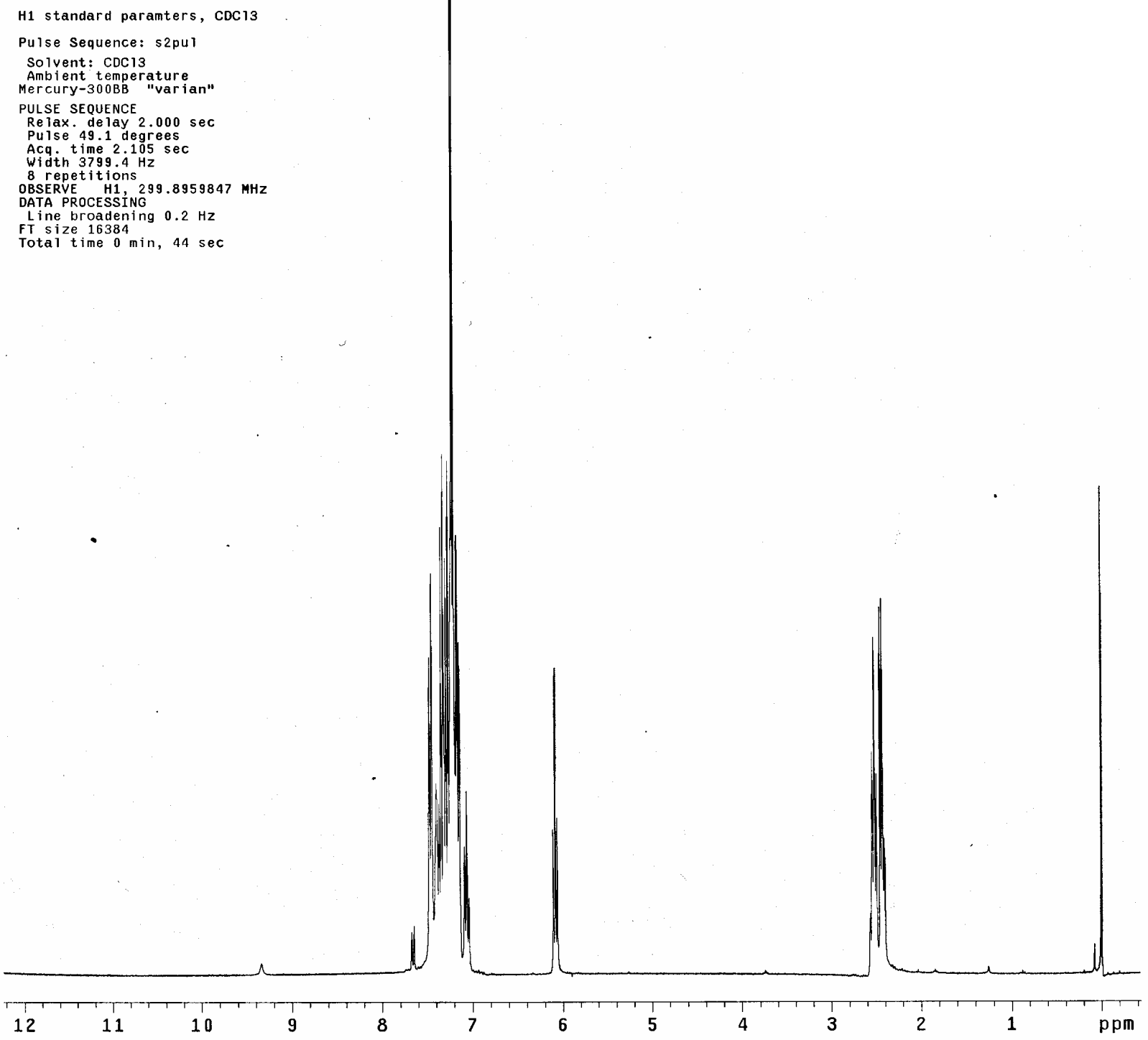


\section{Compound 1a}<smiles>[TeH][TeH]</smiles>

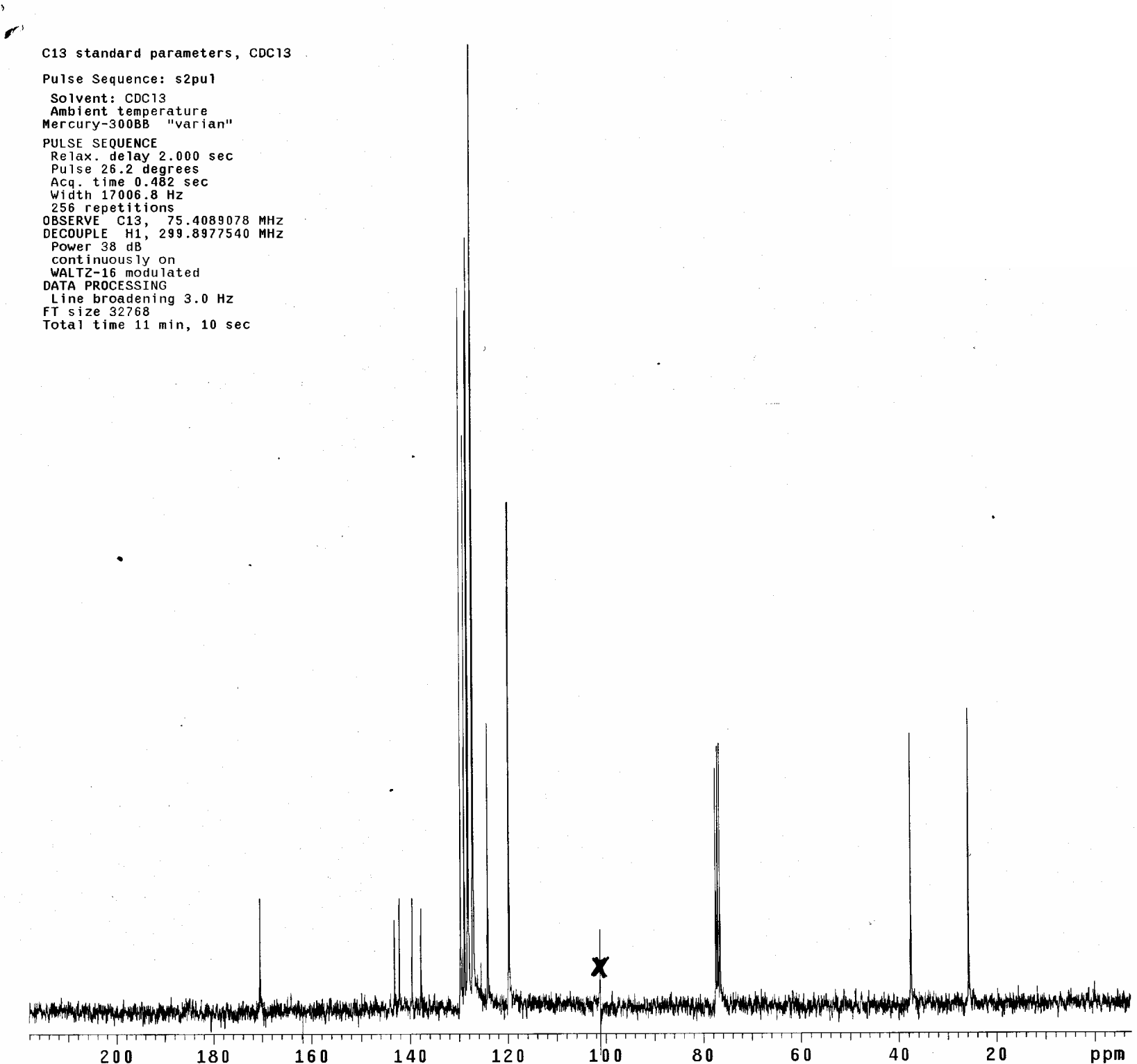




\section{Compound 1b}

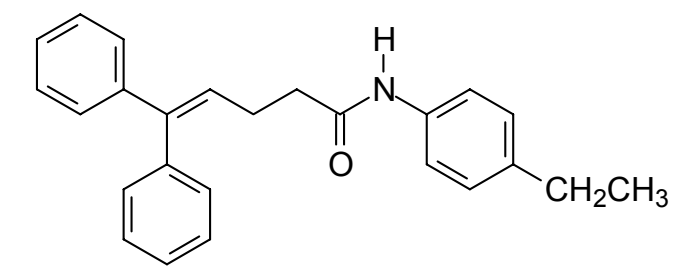

H1 standard parameters, $\mathrm{CDC} 13$

Pulse Sequence: s2pul

Solvent: $\mathrm{CDC}_{13}$

Mercury-300B "varian"

PULSE SEQUENCE
Relax. delay $2.000 \mathrm{sec}$

Relax. delay 2.000

Acq. time $2.105 \mathrm{sec}$

width $3799.4 \mathrm{~Hz}$

OBSERVE H1 $299.8836777 \mathrm{MHz}$

Line broadening $0.2 \mathrm{~Hz}$

$\mathrm{LT}$ size 16384
Total time $0 \mathrm{~min}, 0 \mathrm{sec}$

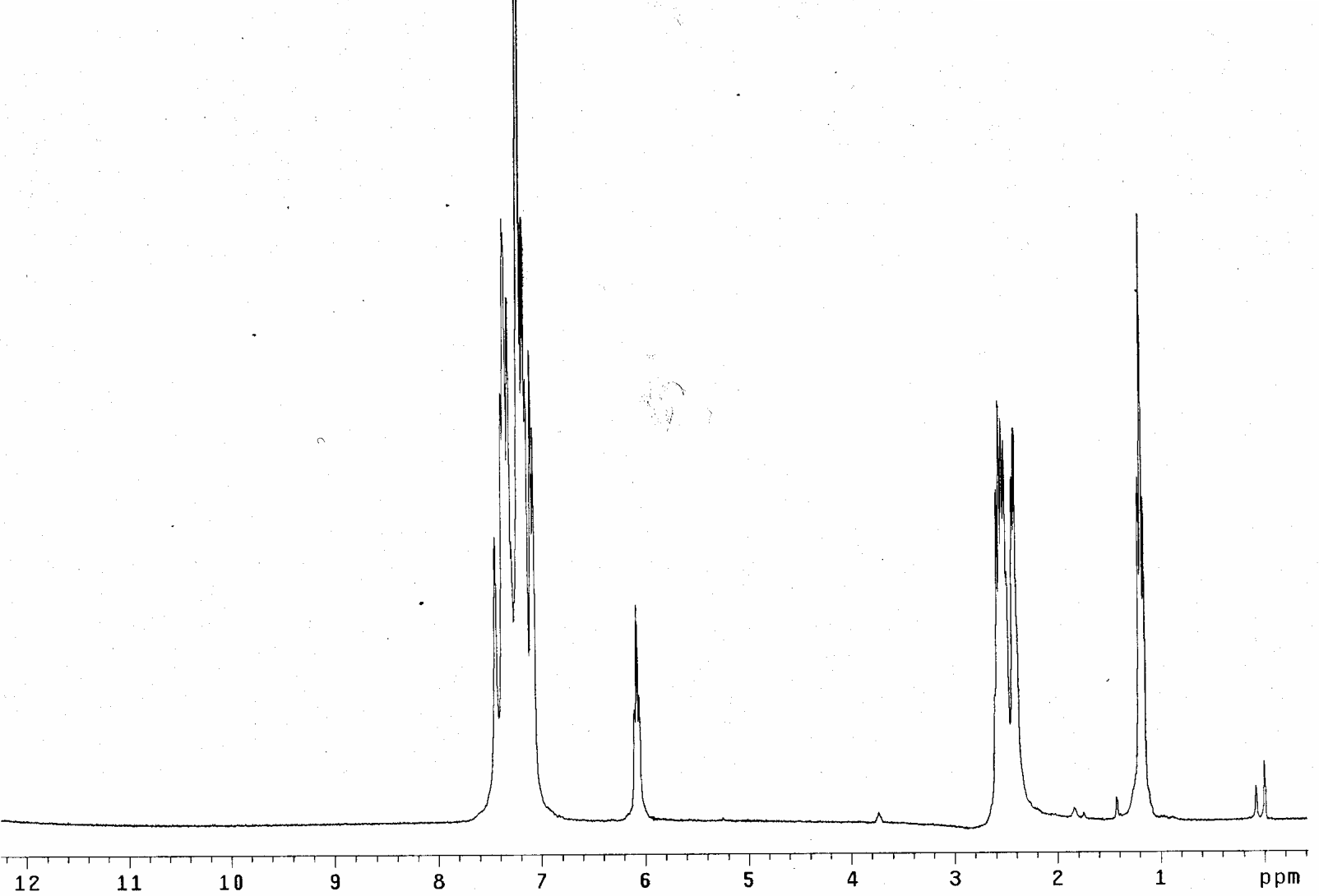




\section{Compound 1b}<smiles>CCc1ccc(NC(=O)CCC=C(c2ccccc2)c2ccccc2)cc1</smiles>

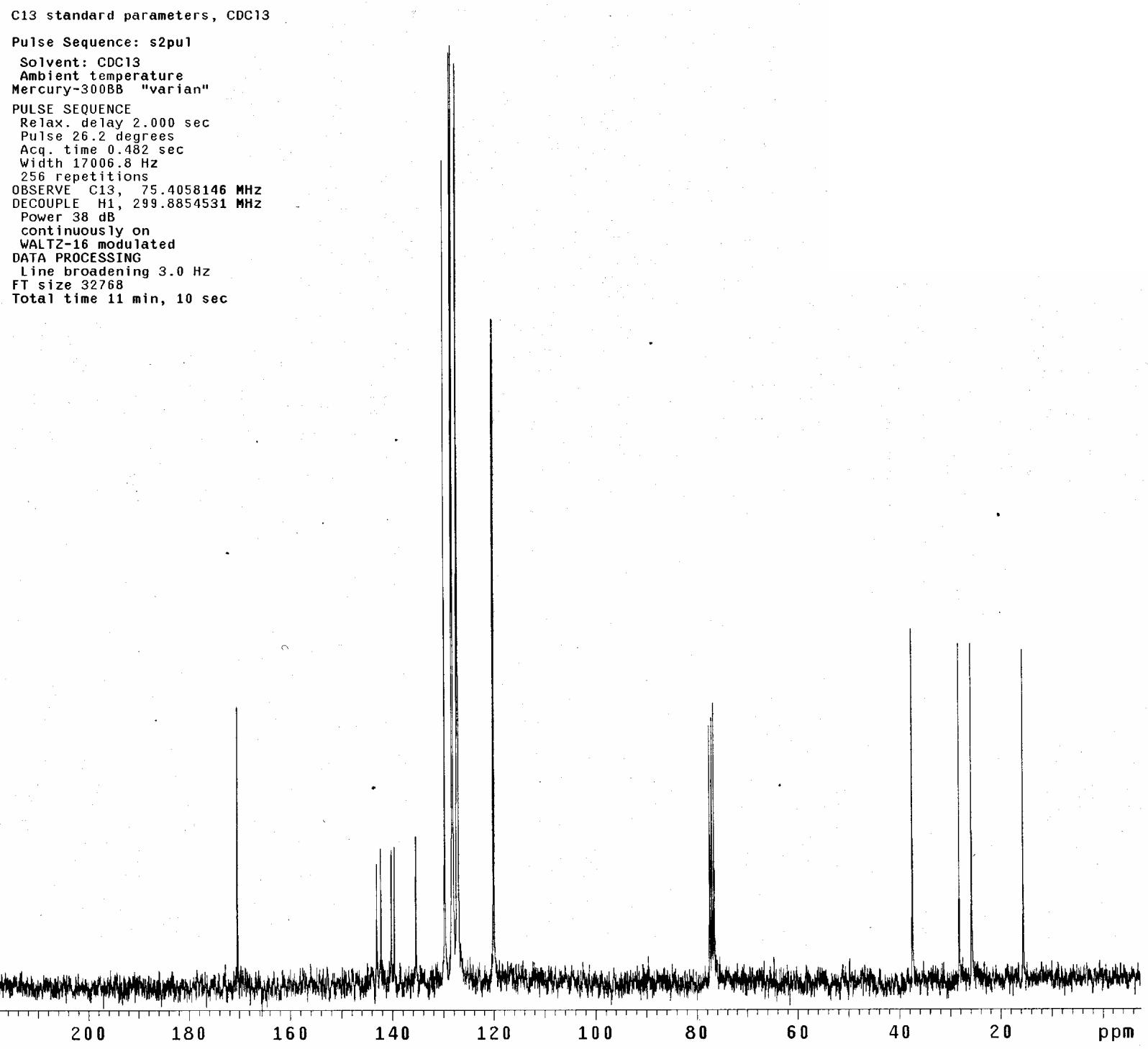


Compound 1c<smiles>O=C(CCC=C(c1ccccc1)c1ccccc1)Nc1ccc(F)cc1</smiles>

Pulse Sequence: s2pul

Solvent: $\mathrm{CDC} 13$

Ambient temperature
Mercury-300BB "varian"

PULSE SEQUENCE

Relax. delay $2.000 \mathrm{sec}$

Pulse 49.1 degrees

Acq. time $2.105 \mathrm{sec}$

8 reptitions

OBSERVE H1, $299.8959847 \mathrm{MHz}$

Line broadening $0.2 \mathrm{~Hz}$

Total time $0 \mathrm{~min}, 44 \mathrm{sec}$

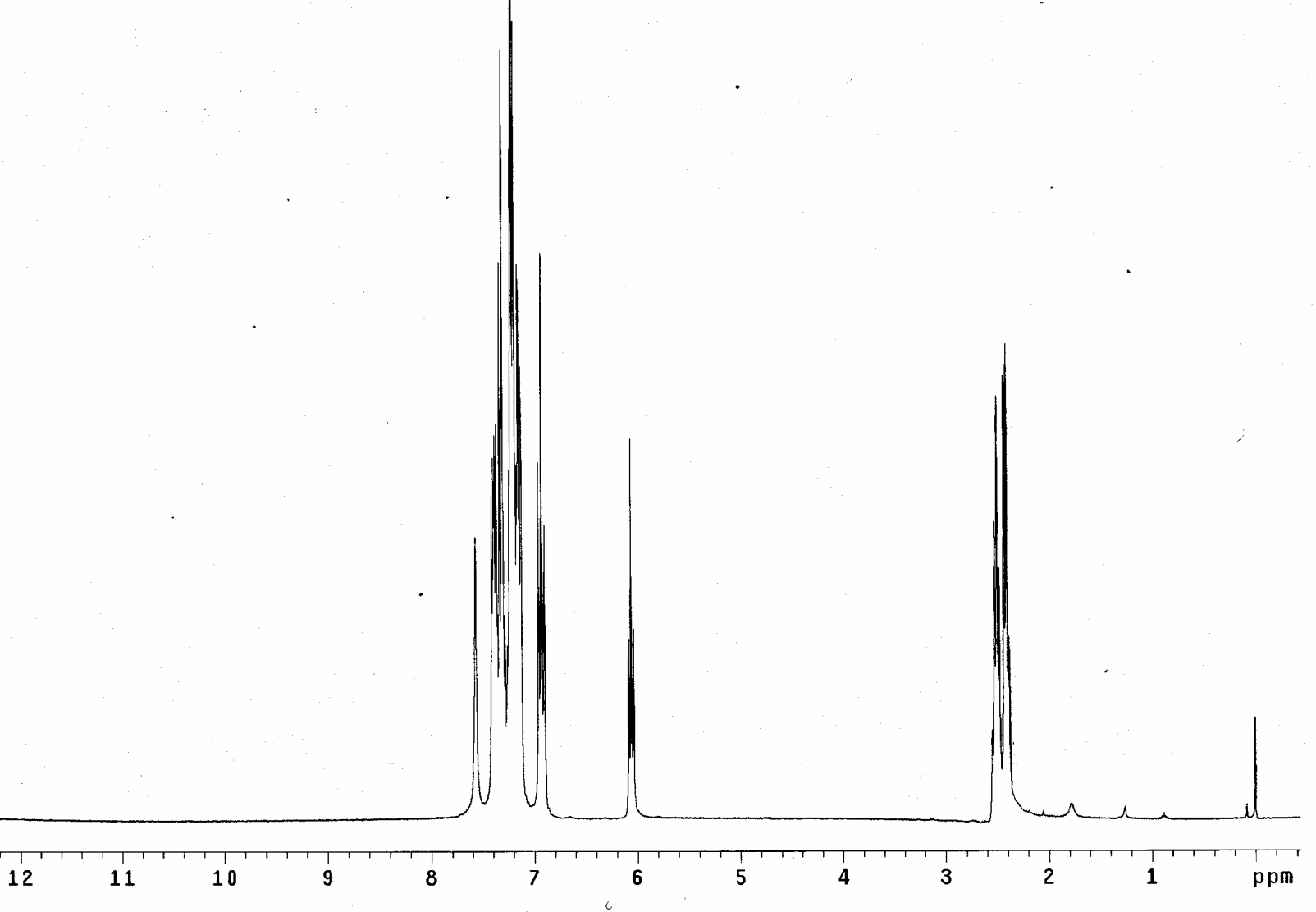




\section{Compound 1c}<smiles>O=C(CCC=C(c1ccccc1)c1ccccc1)Nc1ccc(F)cc1</smiles>

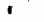

$m$ C13 standard parameters, $\mathrm{CDC}_{13}$

Pulse Sequence: s2pul

Solvent: $\operatorname{CDC} 13$

Amb ent temperature

PULSE SEQUENCE
Relax. de lay $2.000 \mathrm{sec}$

Relax. de lay 2.000
Pulse 26.2 degrees

Acq. time $0.482 \mathrm{sec}$

Width $17006.8 \mathrm{~Hz}$

256 repetitions

OBSERVE C13, $75.4089088 \mathrm{MHZ}$

DECOUPLE H1, 299.8977540 MHZ

Power $38 \mathrm{~dB}$

WALTZ-16 modurated

DATA PROCESSING

Line broadening $3.0 \mathrm{~Hz}$

FT size 32768

Total time $11 \mathrm{~min}, 10 \mathrm{sec}$

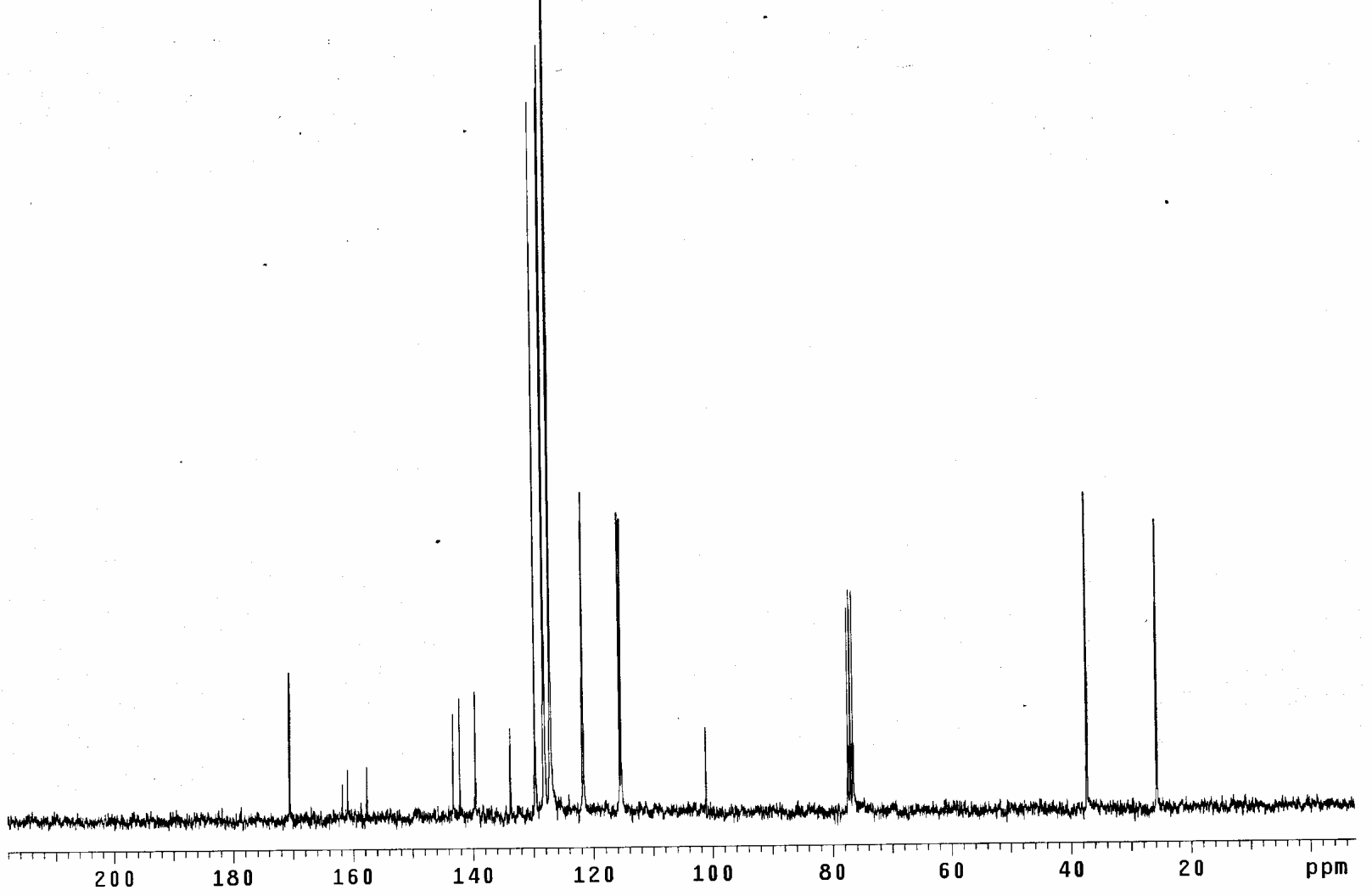




\section{Compound 1d}<smiles>CC(=O)c1ccc(NC(=O)CCC=C(c2ccccc2)c2ccccc2)cc1</smiles>

\section{A}

H1 standard parameters, $\mathrm{CDC} 13$

Pulse Sequence: s2pul

Solvent: $\operatorname{CDC} 13$

Ambient temperature

Mercury-300B B "varian"

PULSE SEQUENCE

Relax. de lay 2.000 sec

Pulse 49.1 degrees

Acq. time $2.105 \mathrm{sec}$

8 repetitions

OBSERVE H1, 299.8836698 MHz

DATA PROCESSİNG

Line broadening $0.2 \mathrm{~Hz}$

FT size 16384
Total time 0 min, 0 sec

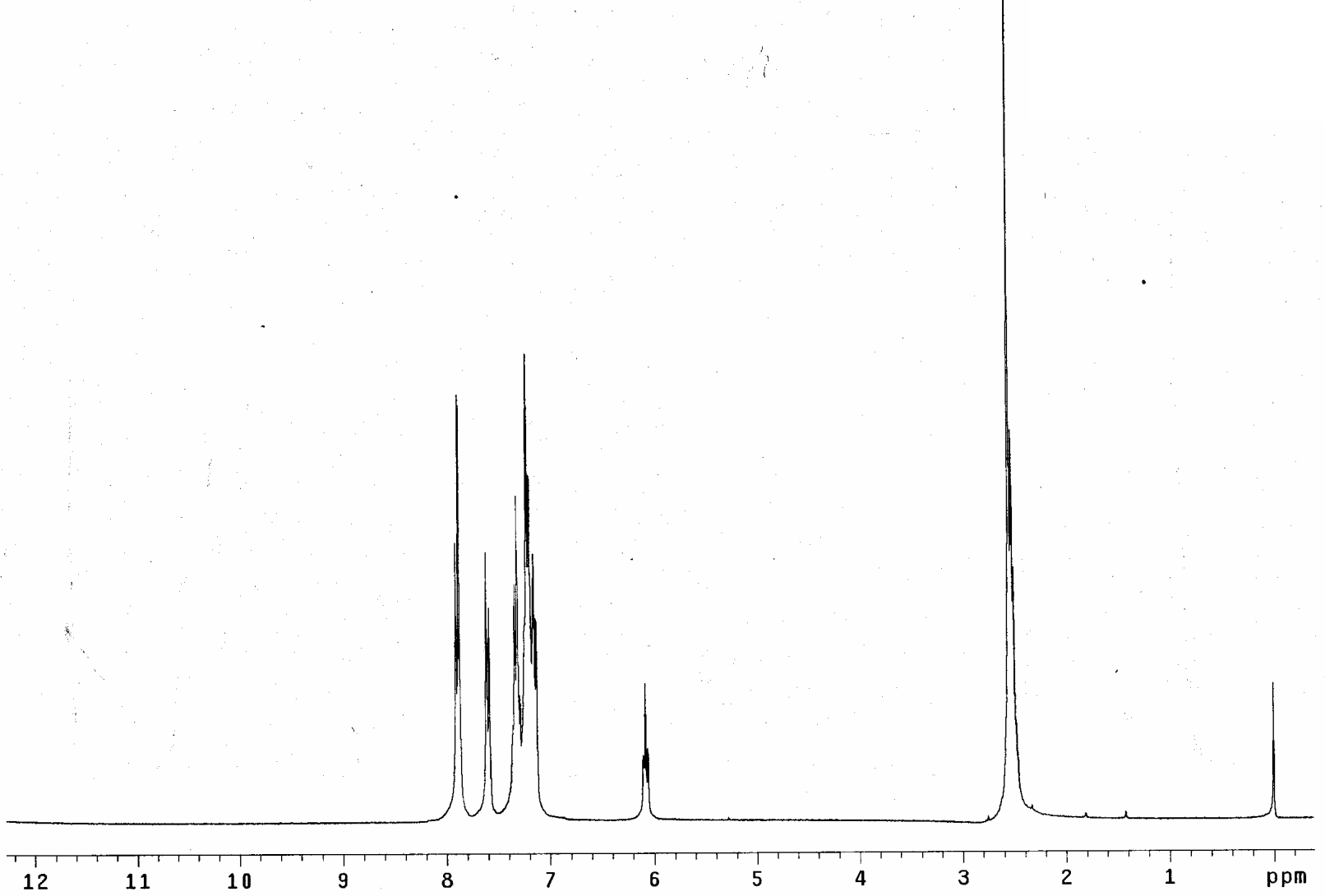

Page S12 


\section{Compound 1d}<smiles>CC(=O)c1ccc(NC(=O)CCC=C(c2ccccc2)c2ccccc2)cc1</smiles>

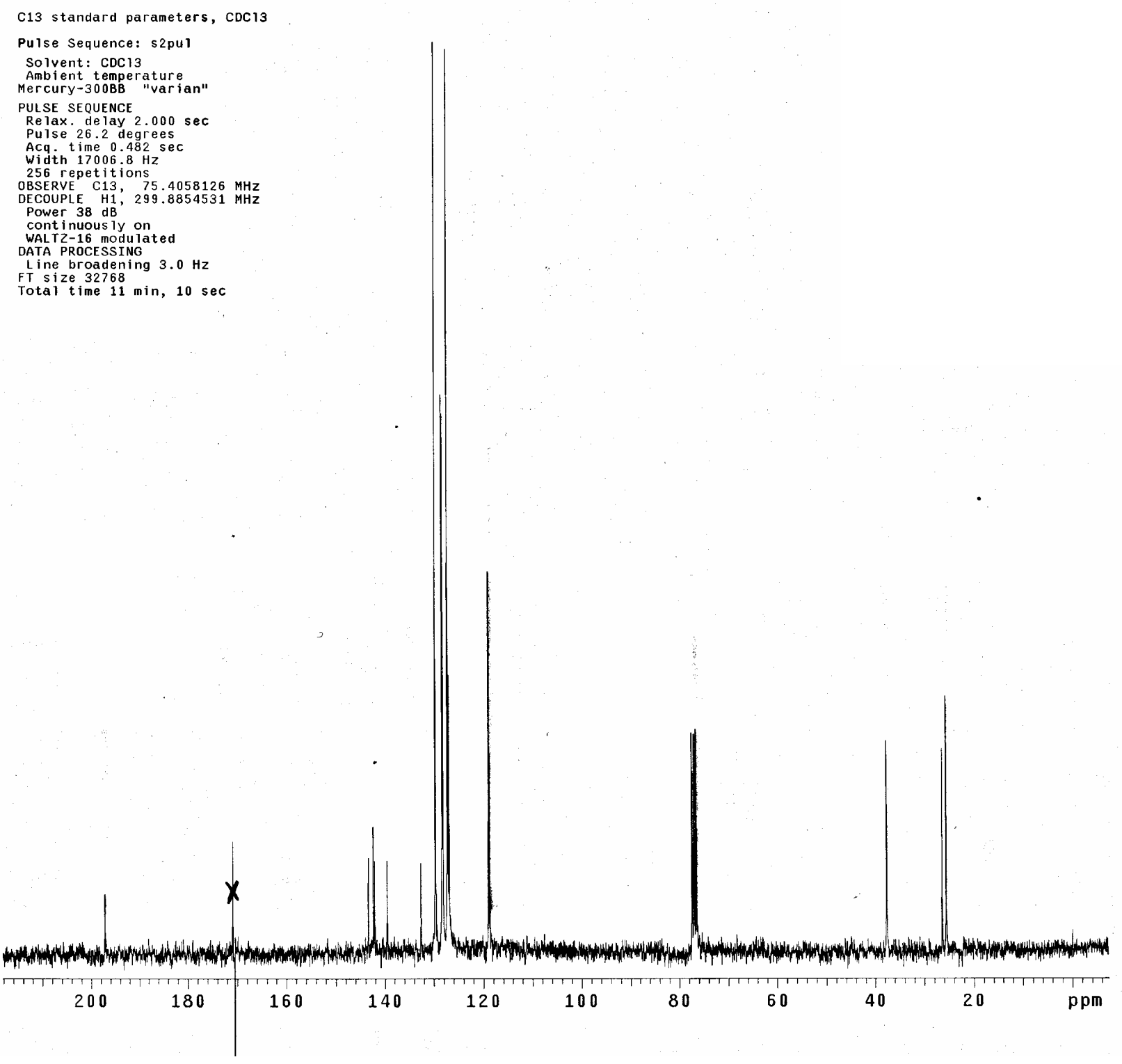




\section{Compound 1e}<smiles>N#Cc1ccc(NC(=O)CCC=C(c2ccccc2)c2ccccc2)cc1</smiles>

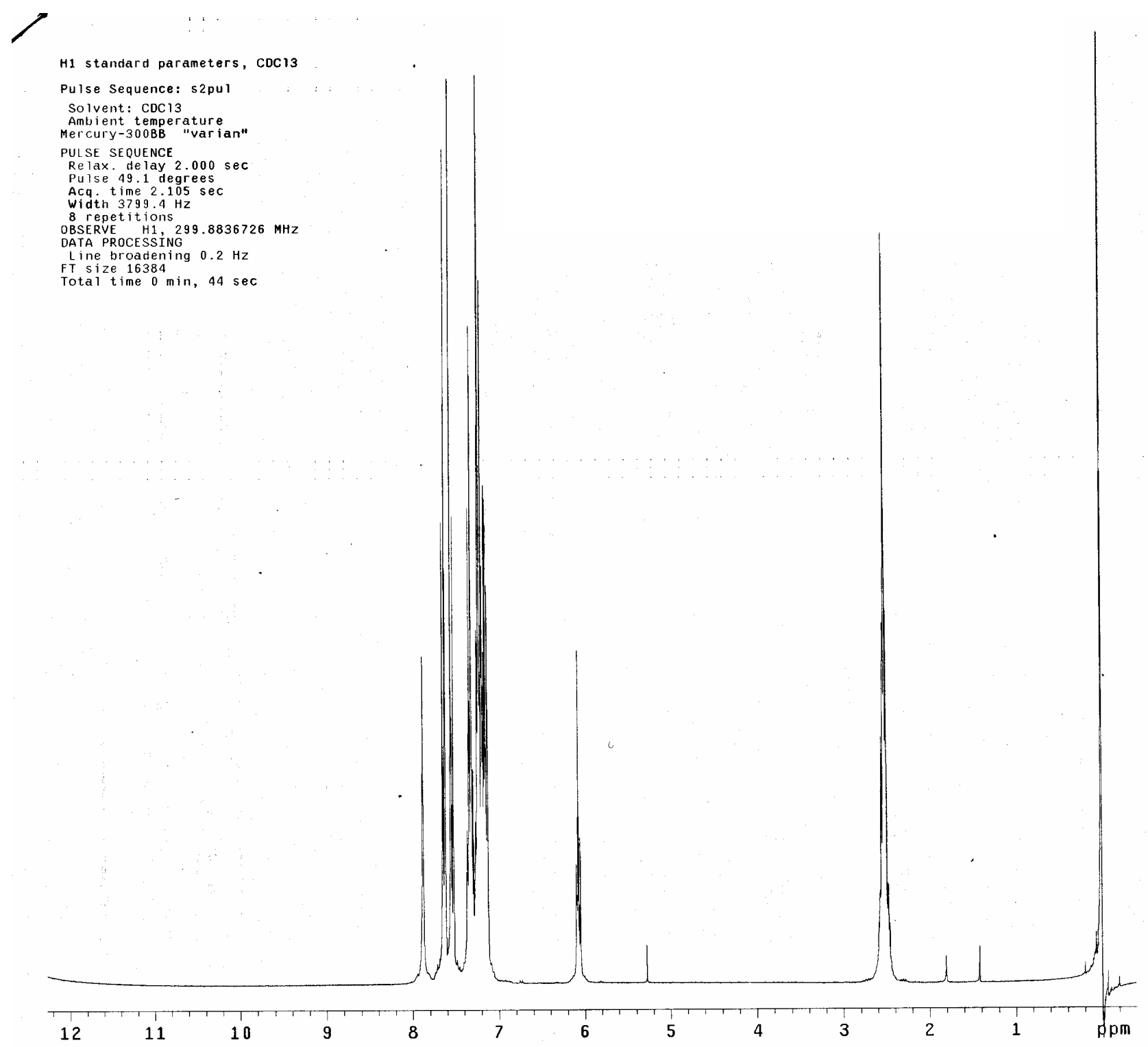




\section{Compound 1e}<smiles>O[Te][Te]</smiles>

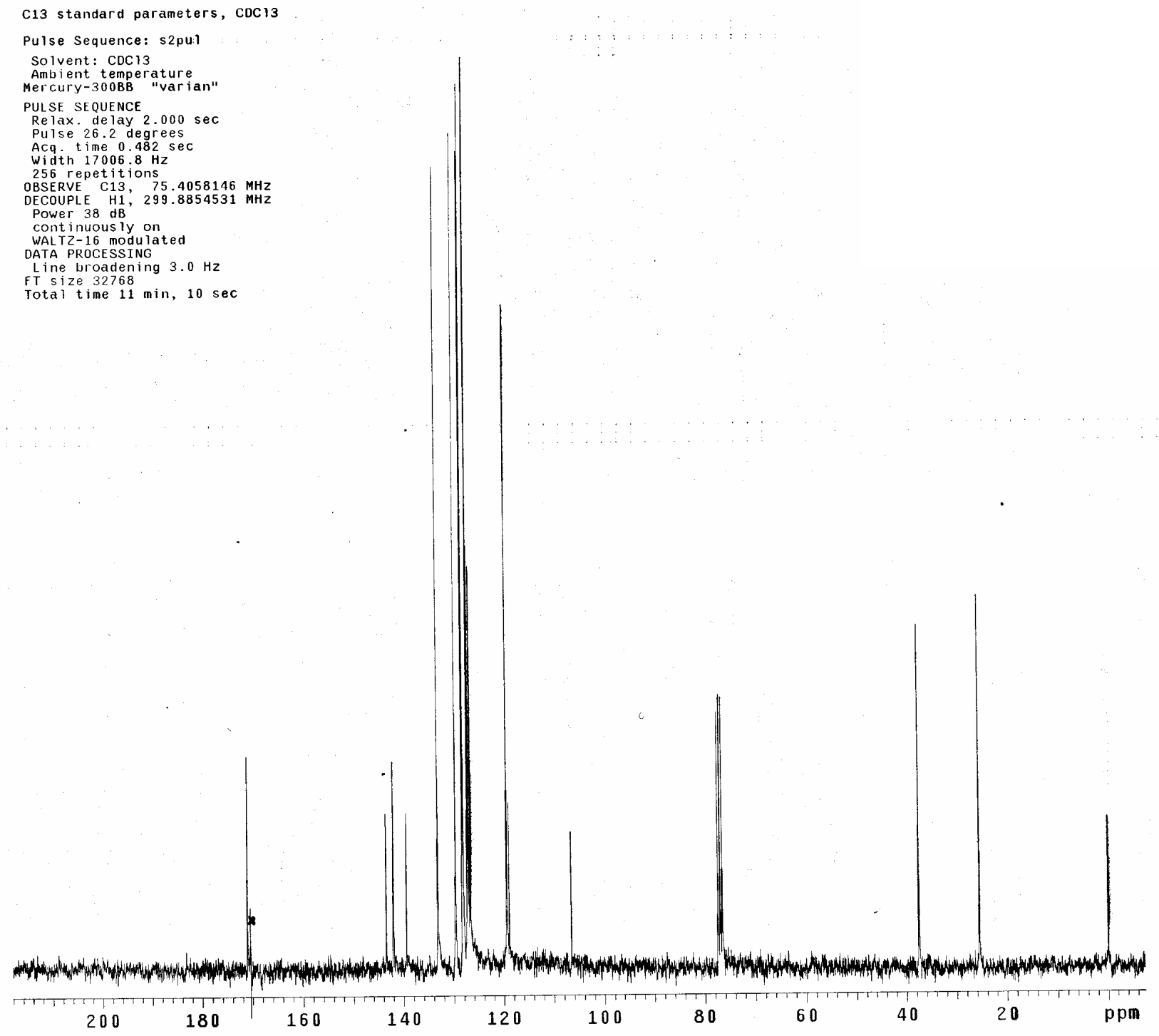




\section{Compound 1f}<smiles>COc1ccc(NC(=O)CCC=C(c2ccccc2)c2ccccc2)cc1</smiles>
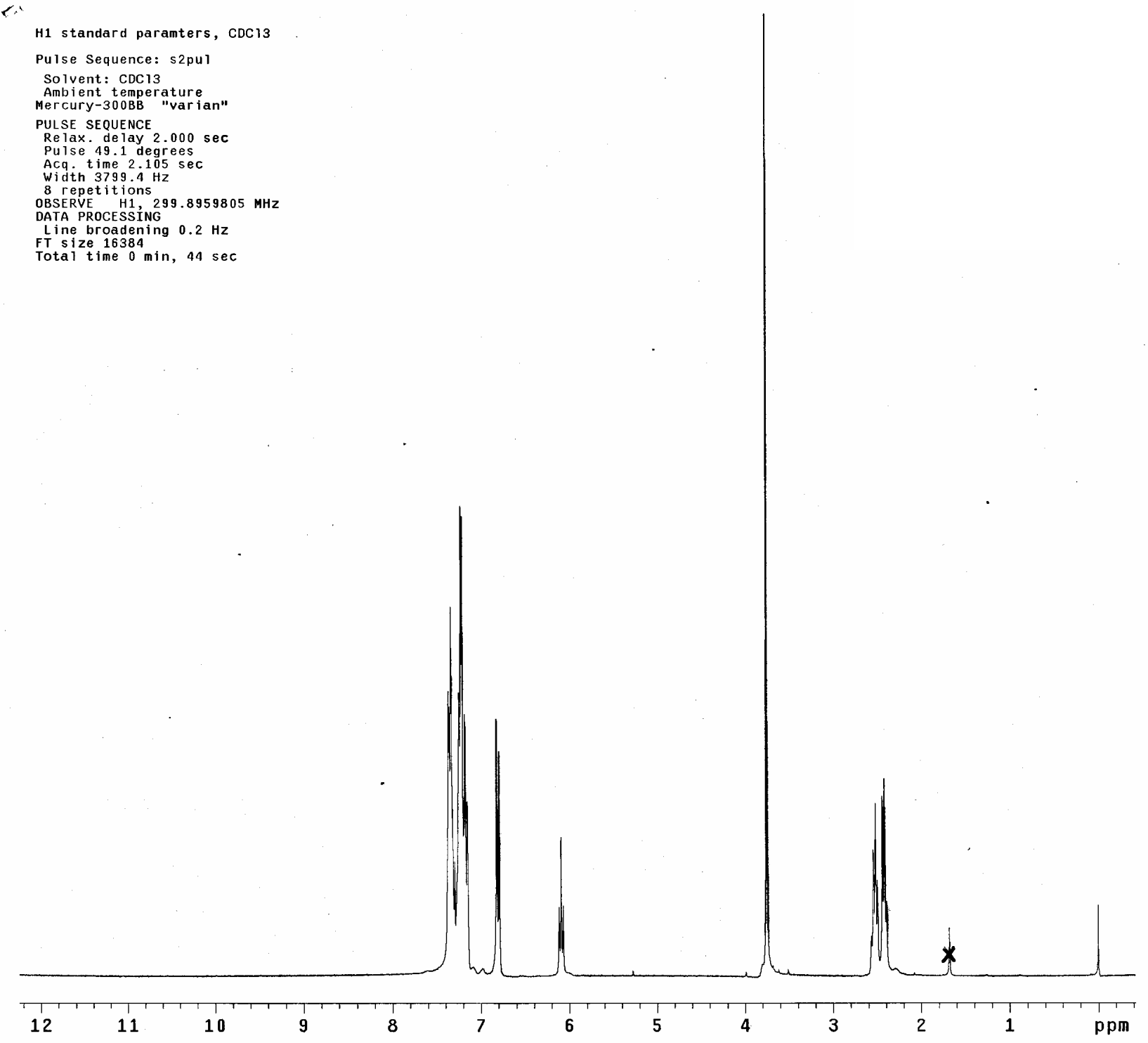


\section{Compound 1f}<smiles>COc1ccc(NC(=O)CCC=C(c2ccccc2)c2ccccc2)cc1</smiles>

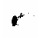

C13 standard parameters, $\mathrm{CDC} 13$

Pulse Sequence: s2pul

Solvent: $\operatorname{CDC} 13$

Mercury-300BB "varian"

PULSE SEOUENCE

Relax. delay $2.000 \mathrm{sec}$

Acq time $0.482 \mathrm{sec}$

Width $17006.8 \mathrm{~Hz}$

256 repetitions

$4089057 \mathrm{MHz}$

DECOUPLE H1, 299.8977540 MHZ

Power 38 dB

WALTZ-16 modulated

DATA PROCESSING

Line broadening $3.0 \mathrm{~Hz}$

Total time $11 \mathrm{~min}, 10 \mathrm{sec}$

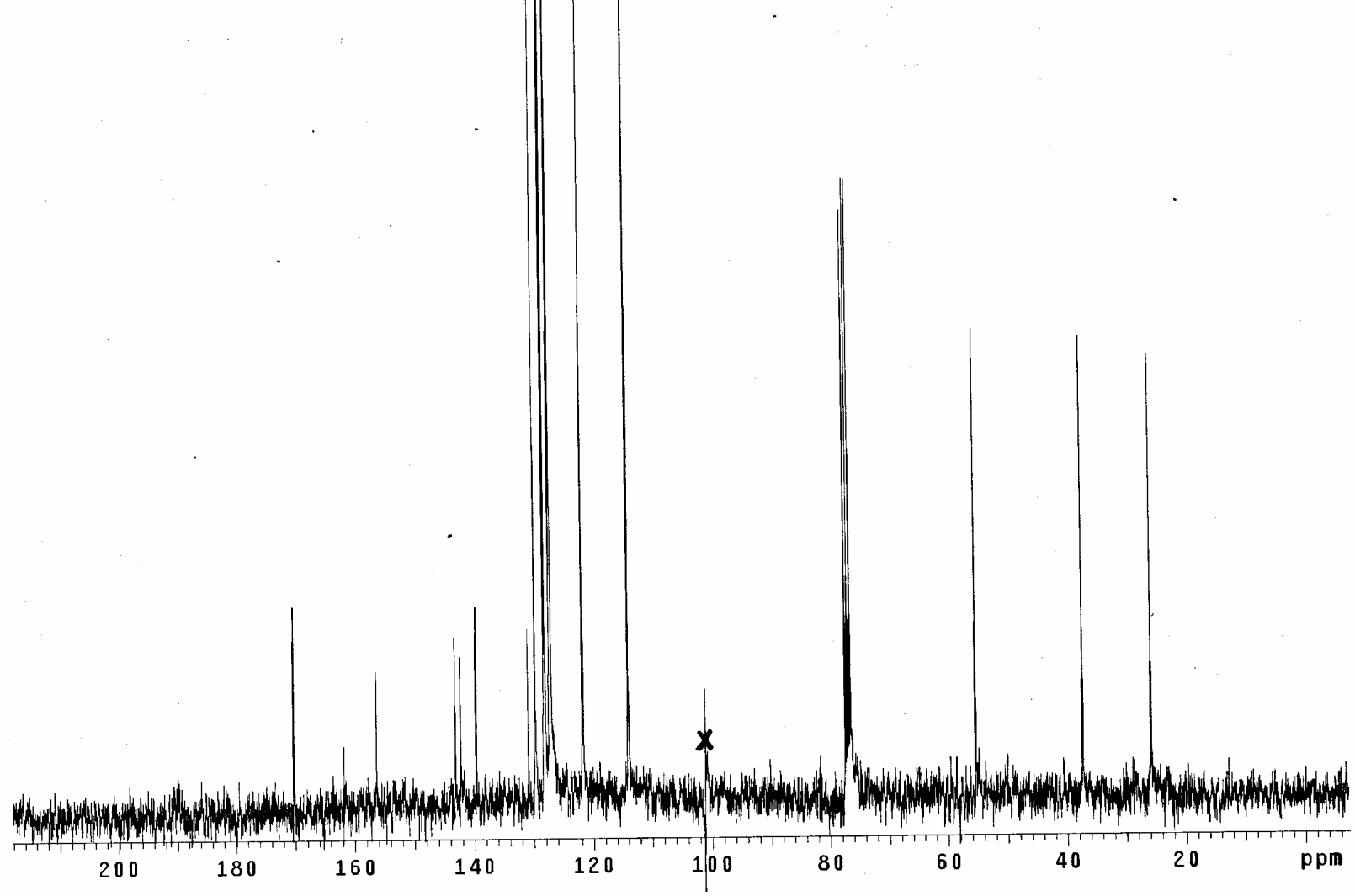




\section{Compound 2a}<smiles>O=C(CCC=C(c1ccccc1)c1ccccc1)N(Sc1ccccc1)c1ccccc1</smiles>
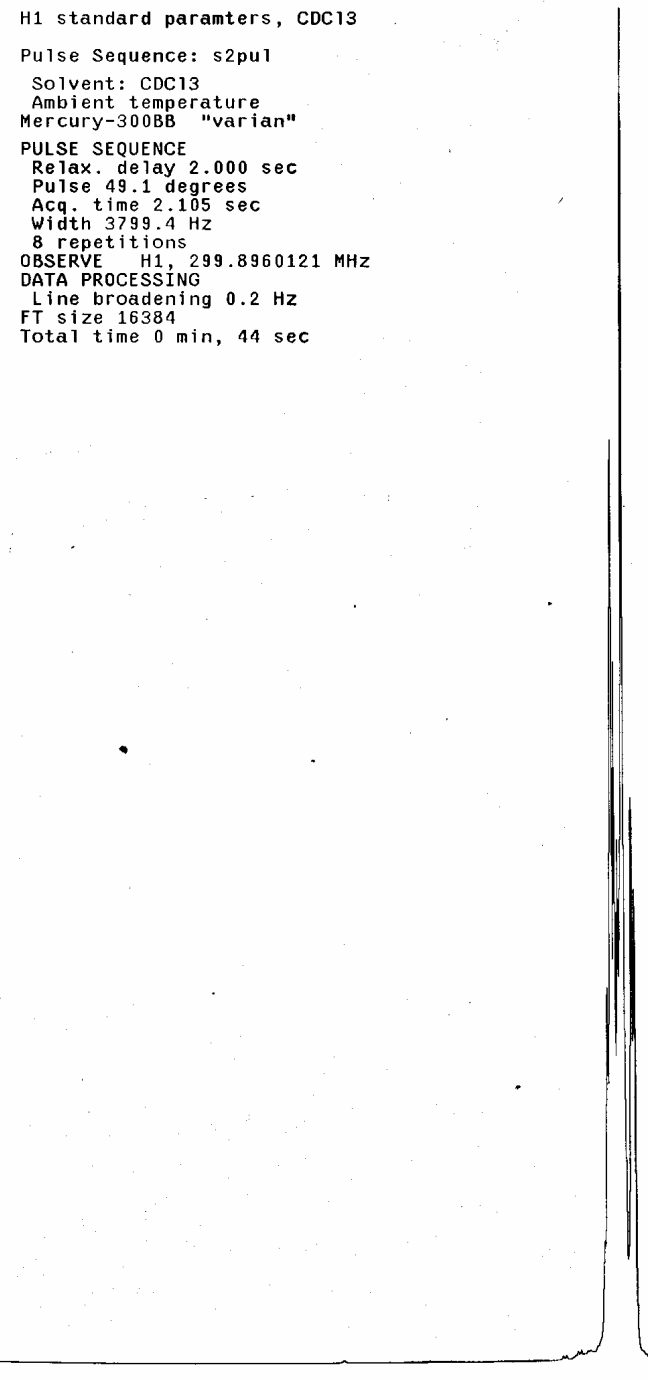


\section{Compound 2a}<smiles>O=C(CCC=C(c1ccccc1)c1ccccc1)N(Sc1ccccc1)c1ccccc1</smiles>
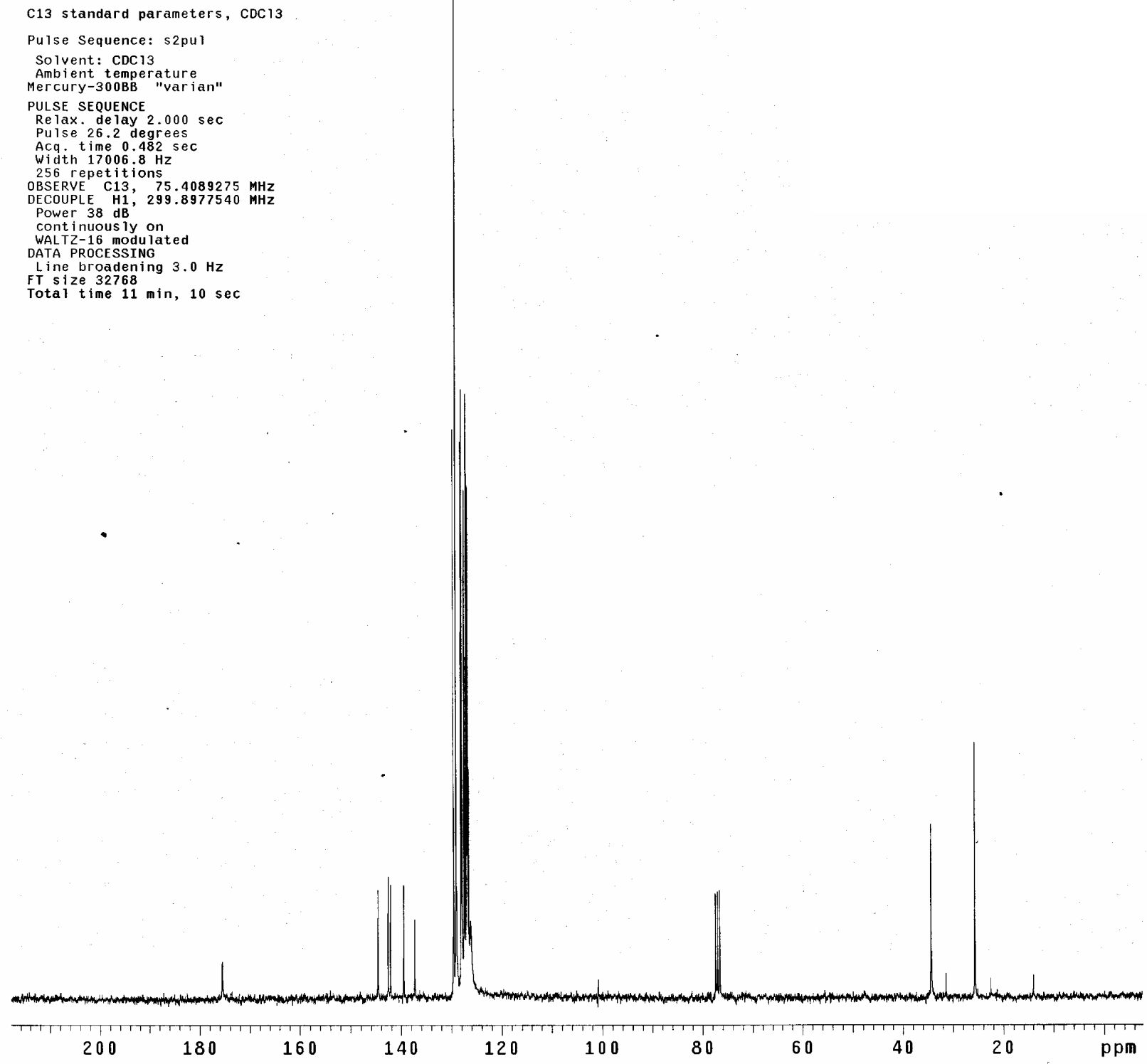


\section{Compound 2b}

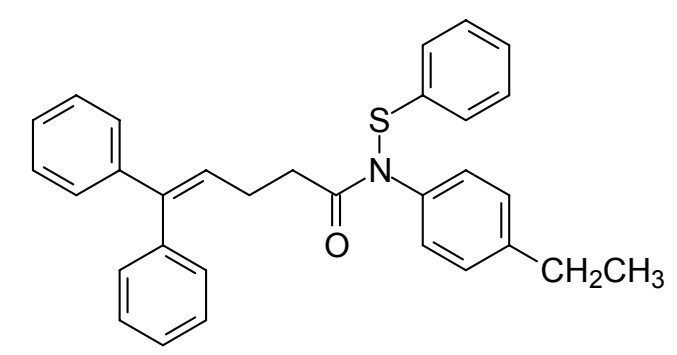

$y$

H1 standard parameters, $\mathrm{COC} 13$

Pulse Sequence: s2pul

Solvent: $\operatorname{CDCl} 13$

Mercury-300BB "vaŕian"

PULSE SEQUENCE

Relax. delay $2.000 \mathrm{sec}$

Acq. time $2.105 \mathrm{sec}$

Width $3799.4 \mathrm{~Hz}$

8 repetitions
OBSERVE H1, 299.8837041 MHZ

OBSERVE H1,

Line broadening $0.2 \mathrm{~Hz}$

Total time 0 min, $0 \mathrm{sec}$ 


\section{Compound 2b}
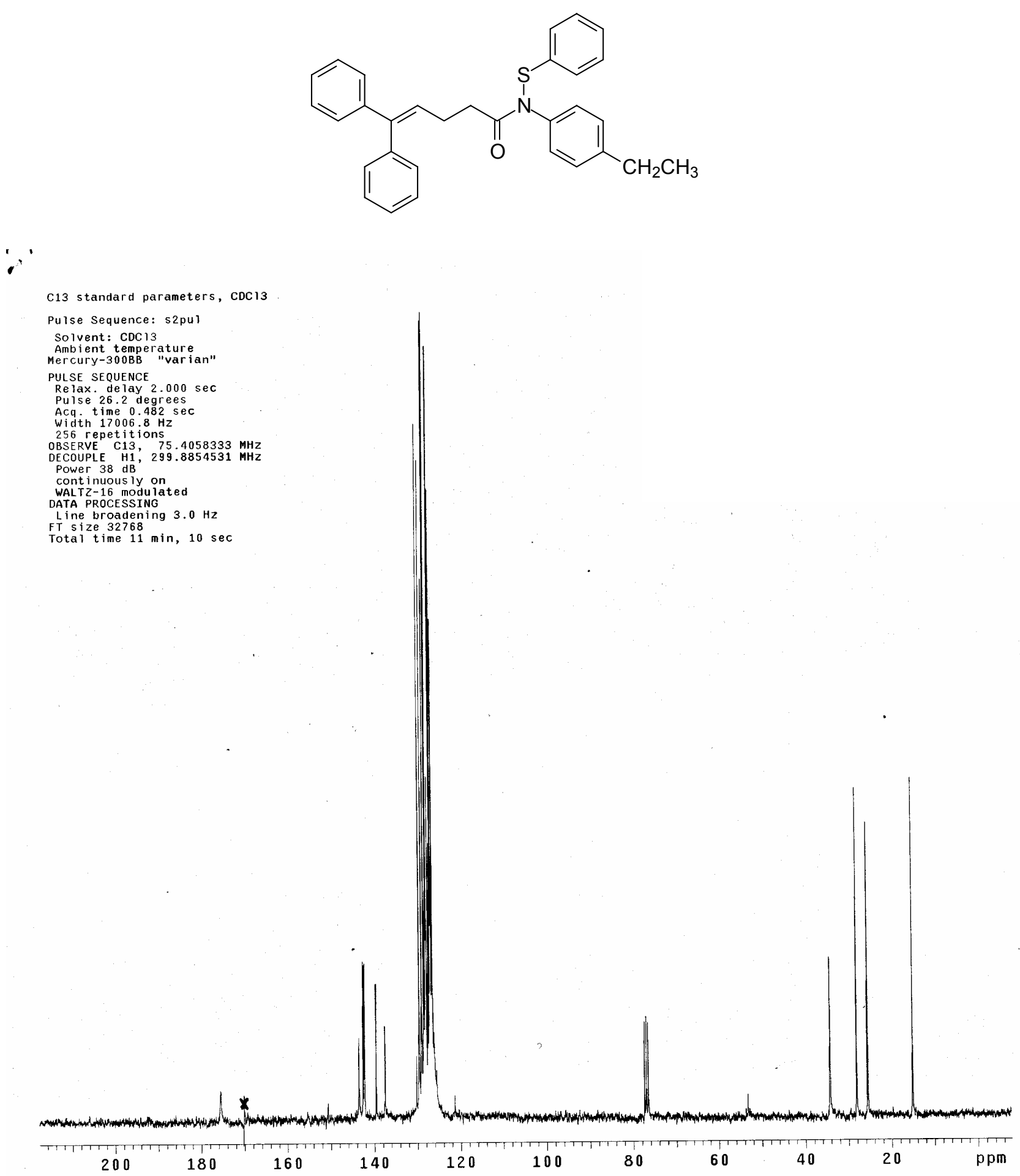


\section{Compound 2c}<smiles>O=C(CCC=C(c1ccccc1)c1ccccc1)N(Sc1ccccc1)c1ccc(F)cc1</smiles>

ง
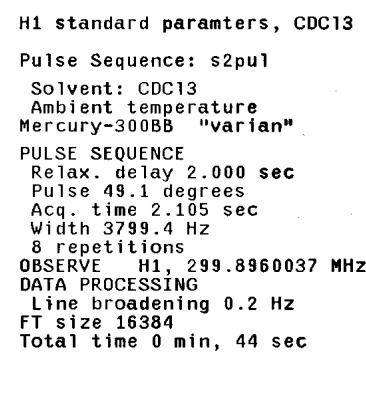


\section{Compound 2c}<smiles>O=C(CCC=C(c1ccccc1)c1ccccc1)N(Sc1ccccc1)c1ccc(F)cc1</smiles>

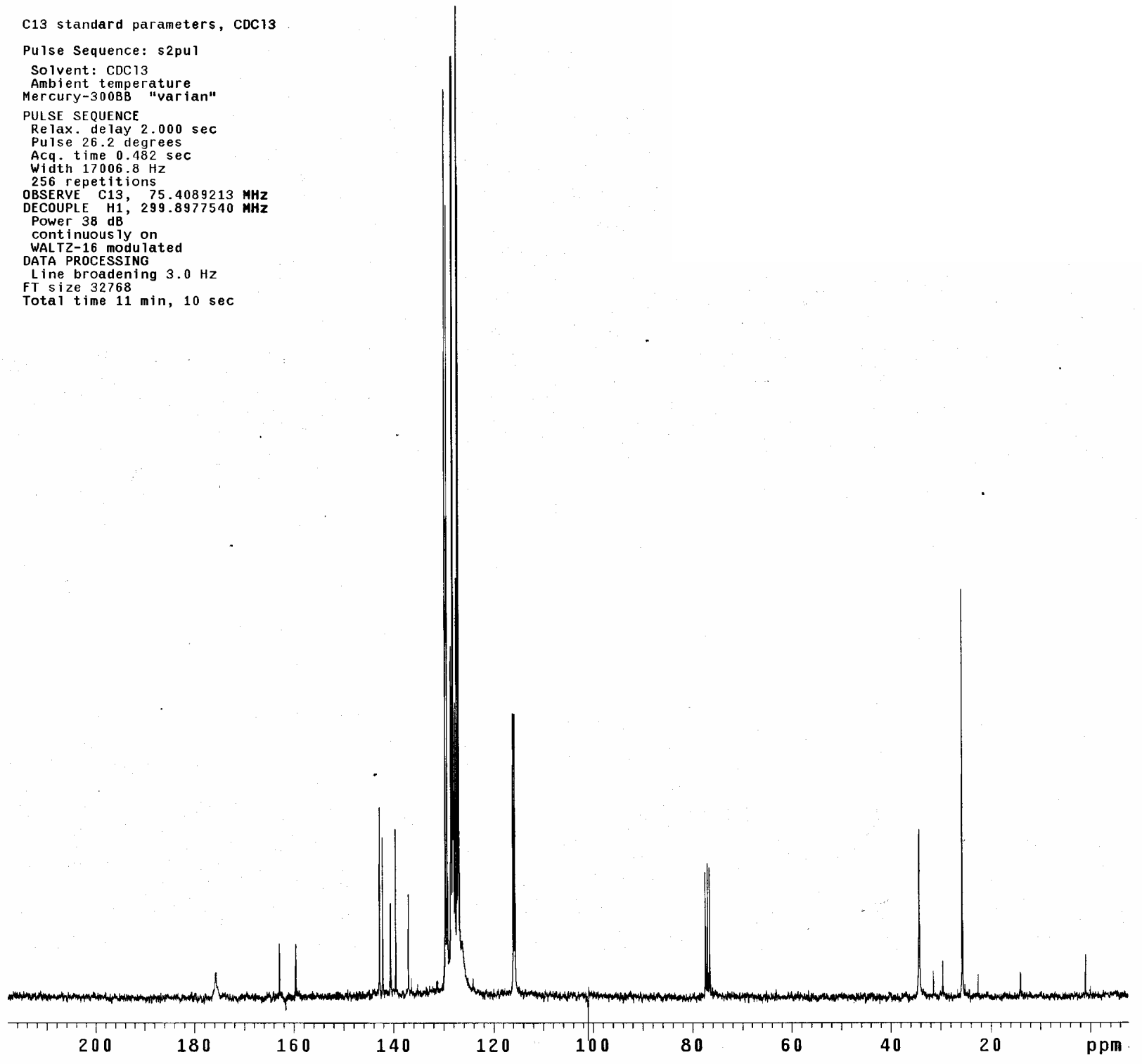


Compound 2d<smiles>CC(=O)c1ccc(N(Sc2ccccc2)C(=O)CCC=C(c2ccccc2)c2ccccc2)cc1</smiles>

H1 standard parameters, $\operatorname{CoCl} 3$

Pulse Sequence: $\$ 2 p u 1$

Solvent: $\mathrm{CDC} 13$

Ambient temperature

Mercury-300BB "varian"

PULSE SEQUENCE

Relax. delay $2.000 \mathrm{sec}$

Pulse 49.1 degrees

Acq. time 2.105 sec

8 repetitions

OBSERVE H1, 299.8836694 MHZ

DATA PROCESSING

Line broadening $0.2 \mathrm{~Hz}$

FT size 16384
Total time $0 \mathrm{~min}, 44 \mathrm{sec}$

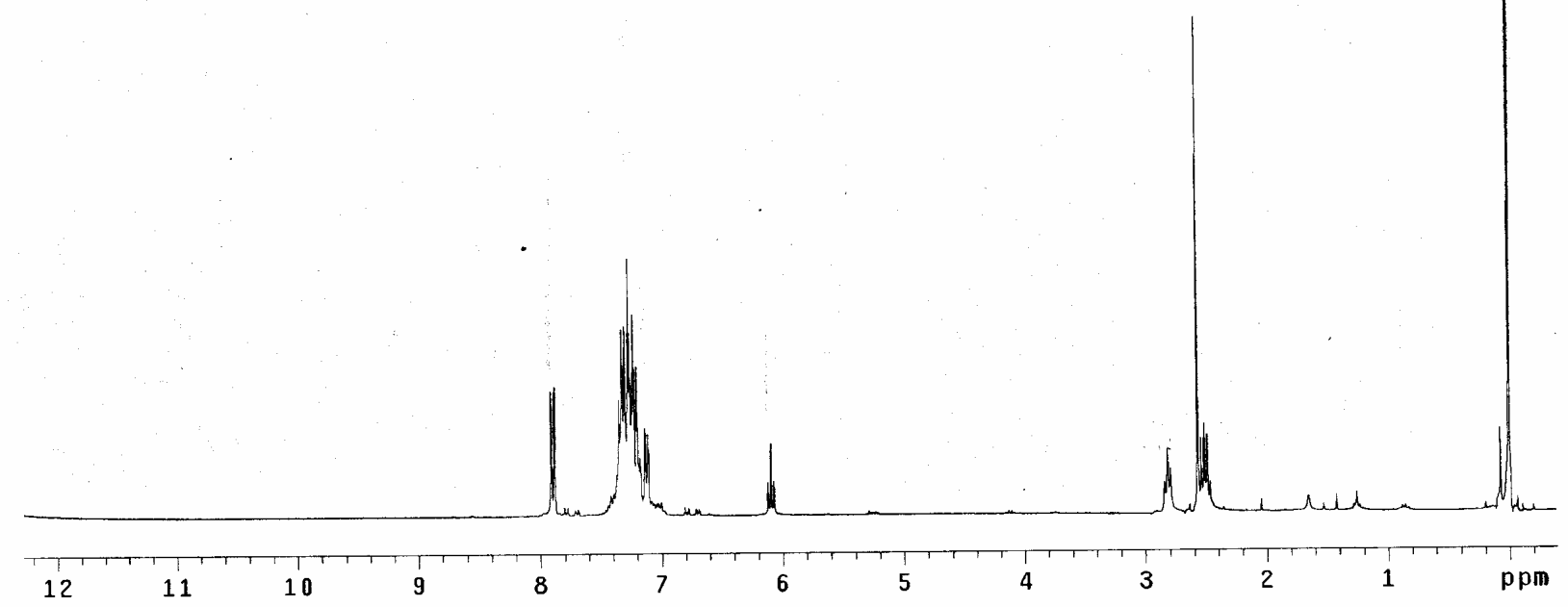

Page S24 


\section{Compound 2d}<smiles>CC(=O)c1ccc(N(Sc2ccccc2)C(=O)CCC=C(c2ccccc2)c2ccccc2)cc1</smiles>
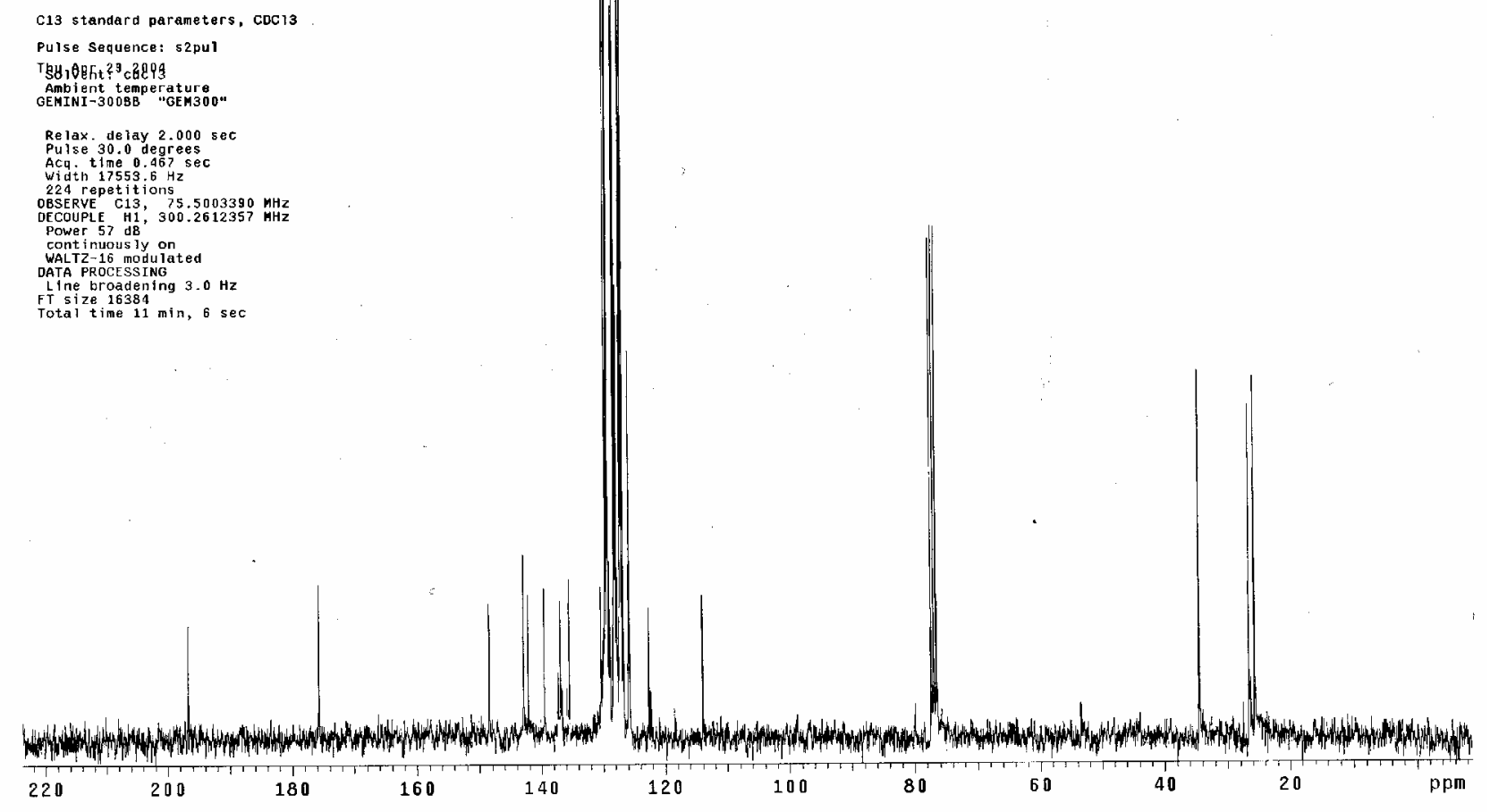


\section{Compound 2e}

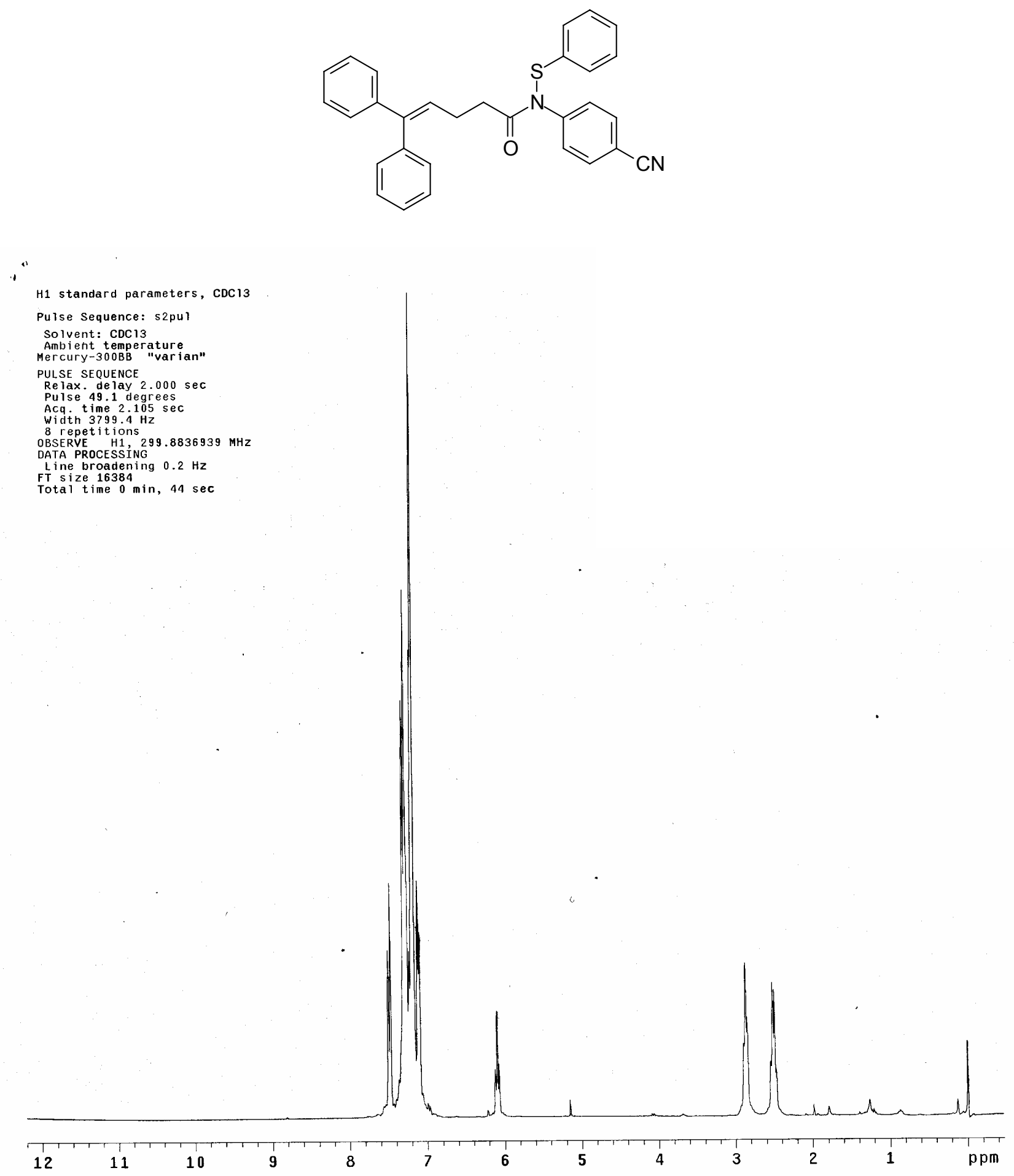




\section{Compound 2e}<smiles>N#Cc1ccc(N(Sc2ccccc2)C(=O)CCC=C(c2ccccc2)c2ccccc2)cc1</smiles>

$\therefore$

C13 standard parameters, $\operatorname{CDC} 13$

Pulse Sequence: s2pul

Solvent: $\operatorname{CDC13}$

Ambient temperature
Mercury-300B "varian"

PULSE SEQUENCE

Relax. delay $2.000 \mathrm{sec}$

Pulse 26.2 degrees

Acq. time $0.482 \mathrm{sec}$

Width $17006.8 \mathrm{~Hz}$
144 repetitions

144 repetitions 4058385 MHZ

DECOUPLE H1, $299.8854531 \mathrm{MHZ}$

Power $38 \mathrm{~dB}$

cont inuous ly on

WALTZ-16 modulated

DATA PROCESSING

Line broadening $3.0 \mathrm{~Hz}$

Total time $11 \mathrm{~min}, 10 \mathrm{sec}$

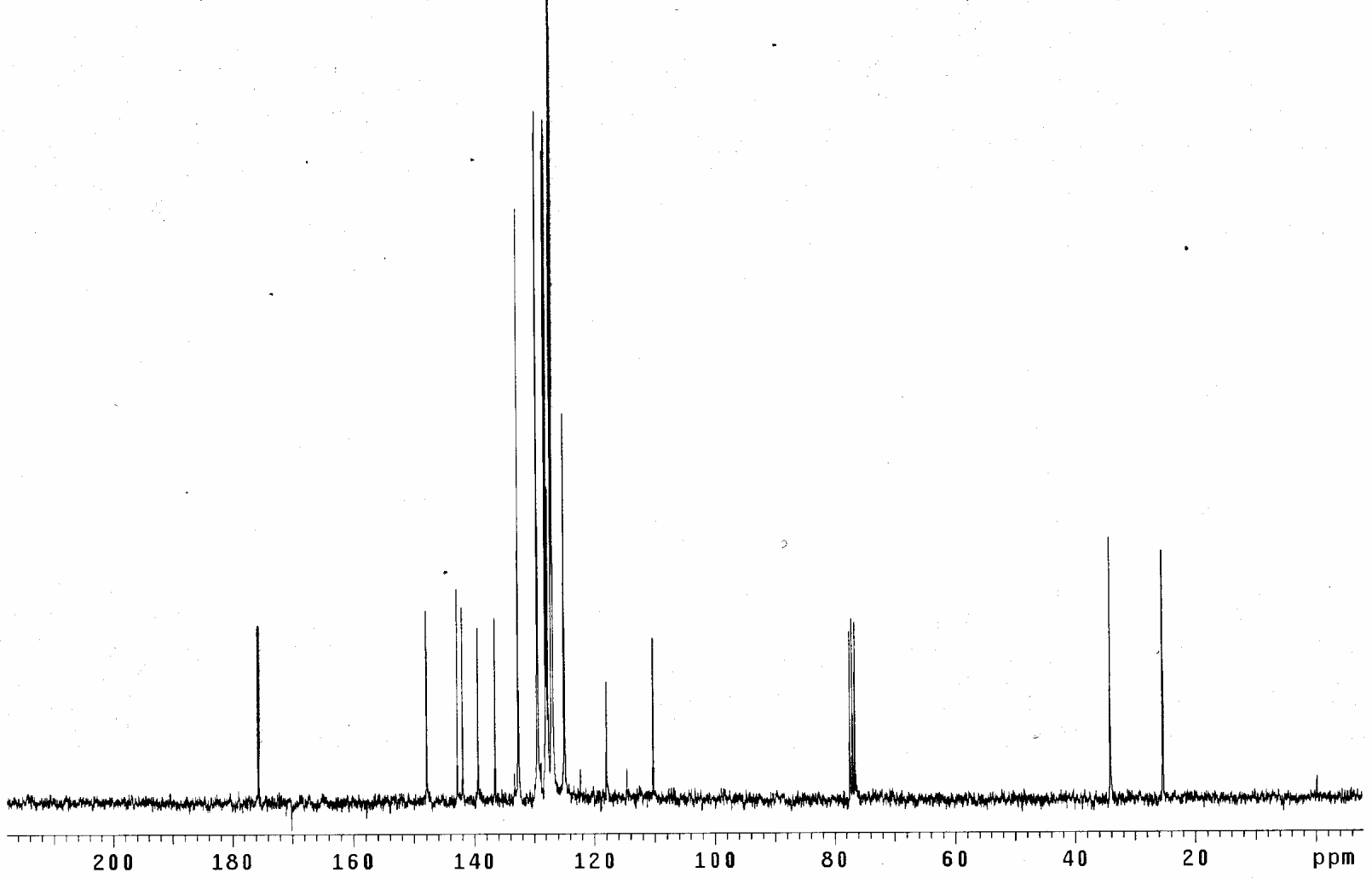




\section{Compound 2f}

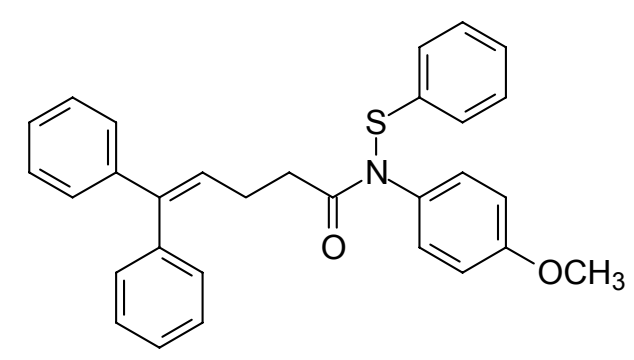

I

H1 standard parameters, $\operatorname{CoC} 13$

Pulse Sequence: s2pur

Solvent: $\operatorname{CDC} 13$

Mercury-300BB "varian"

PULSE SEQUENCE

PULSE SEQUENCE
Relax. delay $2.000 \mathrm{sec}$

Rulax. de lay 2.000

Acq. time 2.105 sec

Width $3799.4 \mathrm{~Hz}$

8 repetitions

OBSERVE H1, 299.8836865
DATA PROCESSING

Line broadening $0.2 \mathrm{~Hz}$

Total time $0 \mathrm{~min}, 44 \mathrm{sec}$

13

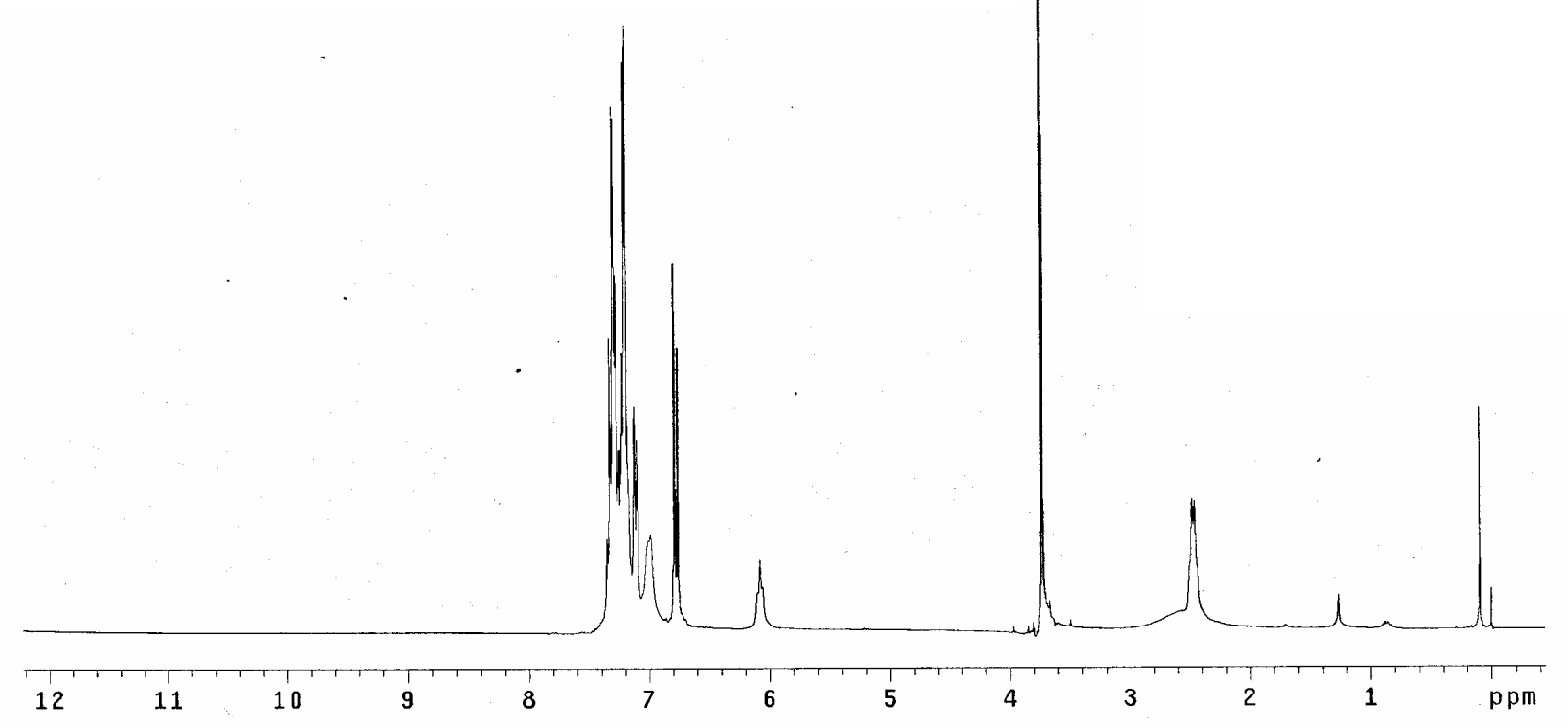

Page S28 


\section{Compound 2f}
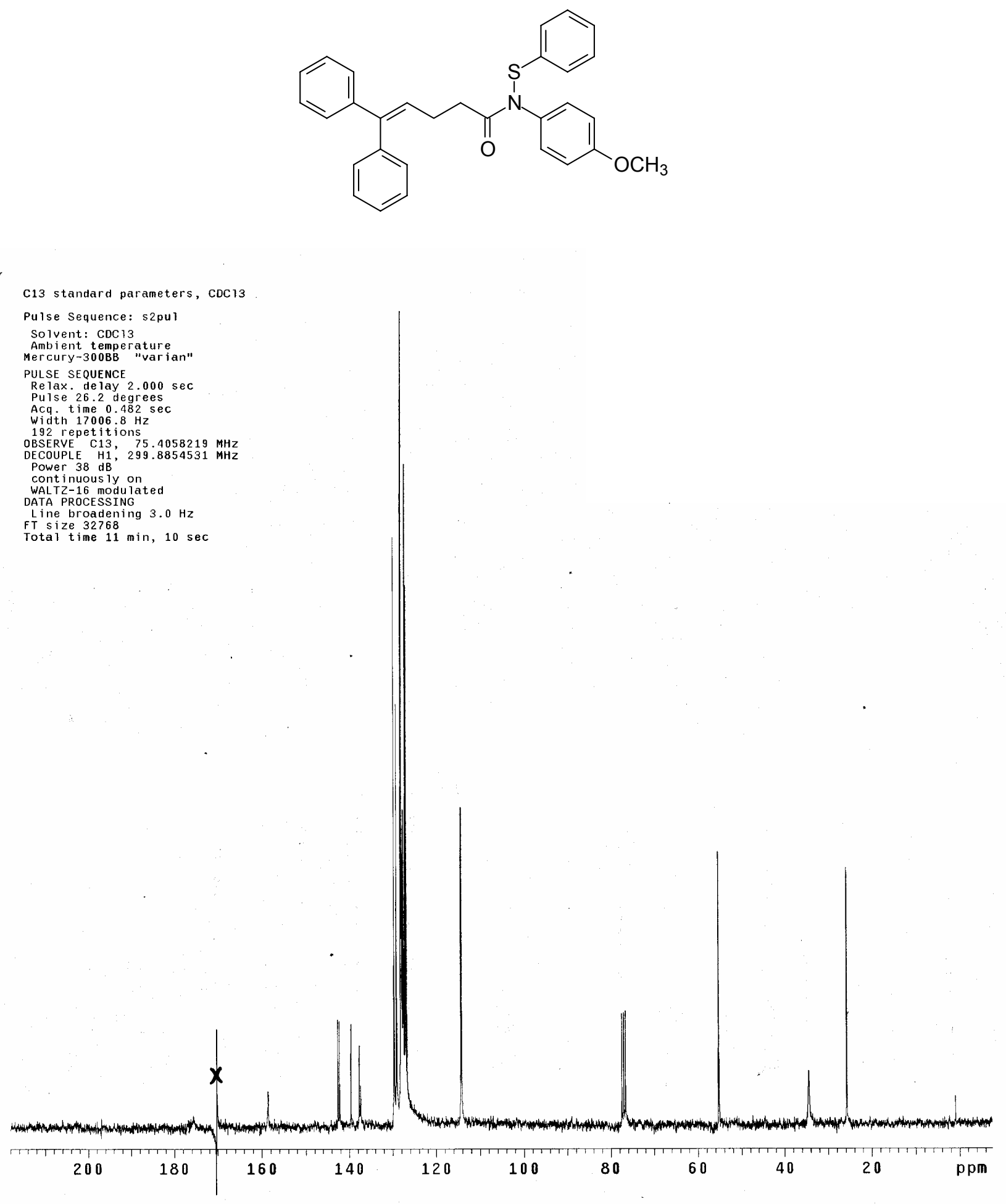
Compound 5a
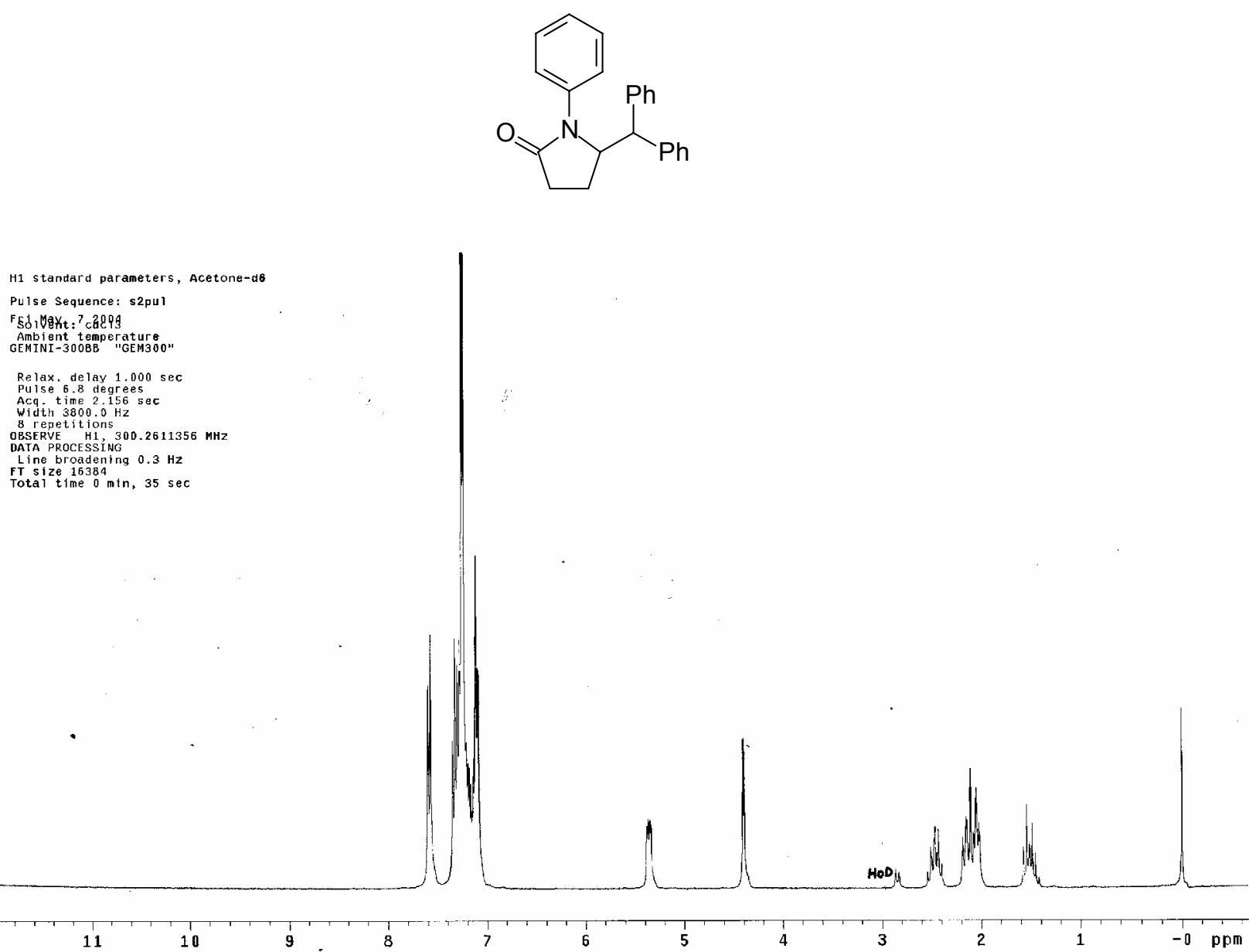


\section{Compound 5a}
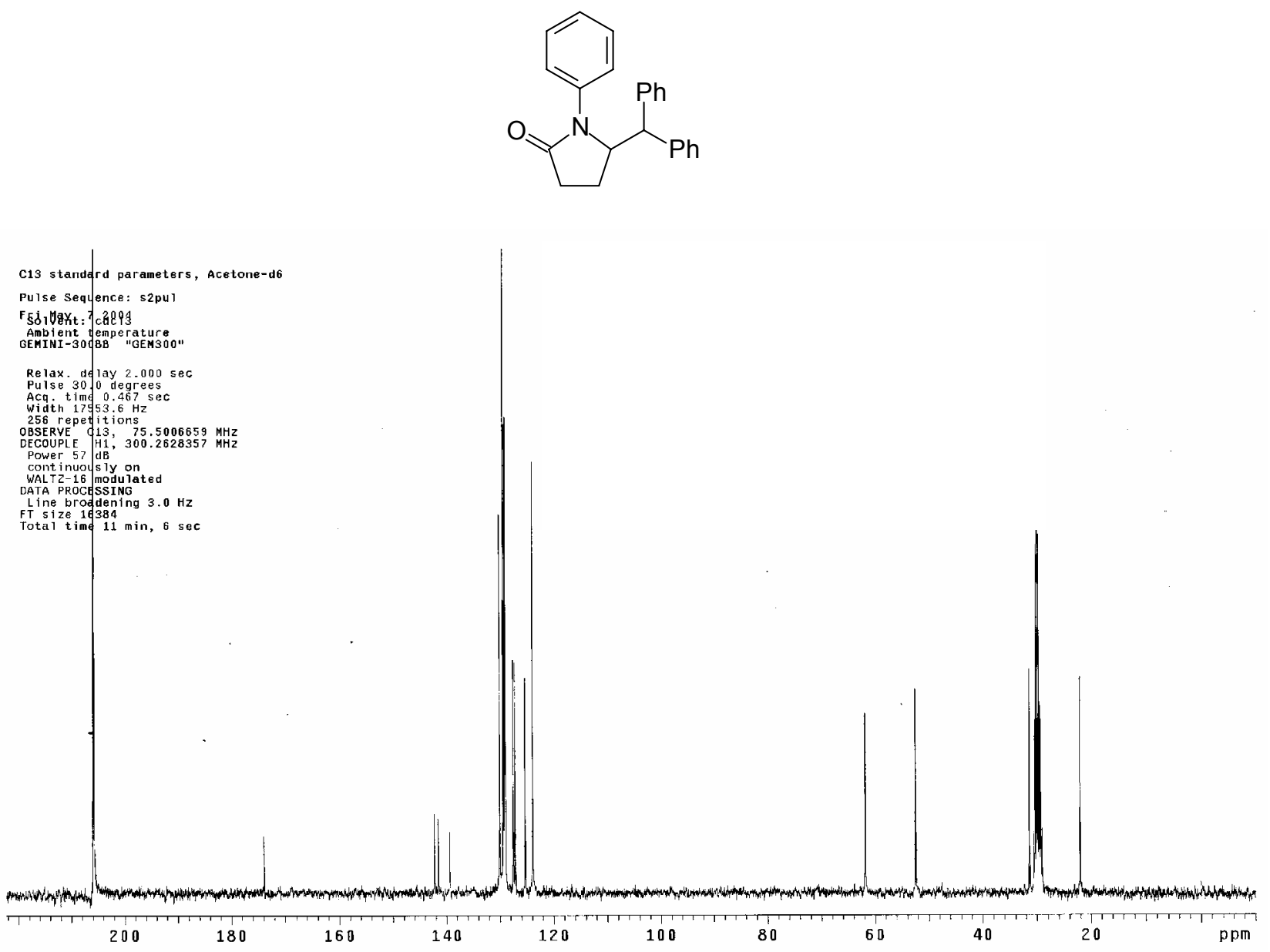
Compound 5b

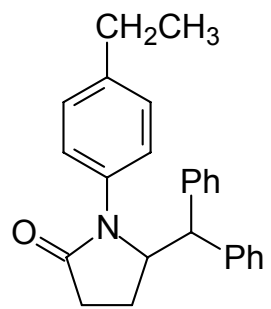

$\%$

H1 standard parameters, Acetone-d6

Pulse Sequence: $52 \mathrm{pul}$

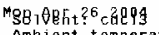

Ambient temperature
GEMTNT-300BB "GEM300"

Re lax. delay $1.000 \mathrm{sec}$

Acy time $2.156 \mathrm{sec}$

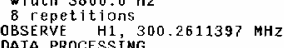

Line broadening $0.3 \mathrm{~Hz}$

FT size 16384
Total t tme $0 \mathrm{~min}, 35 \mathrm{sec}$
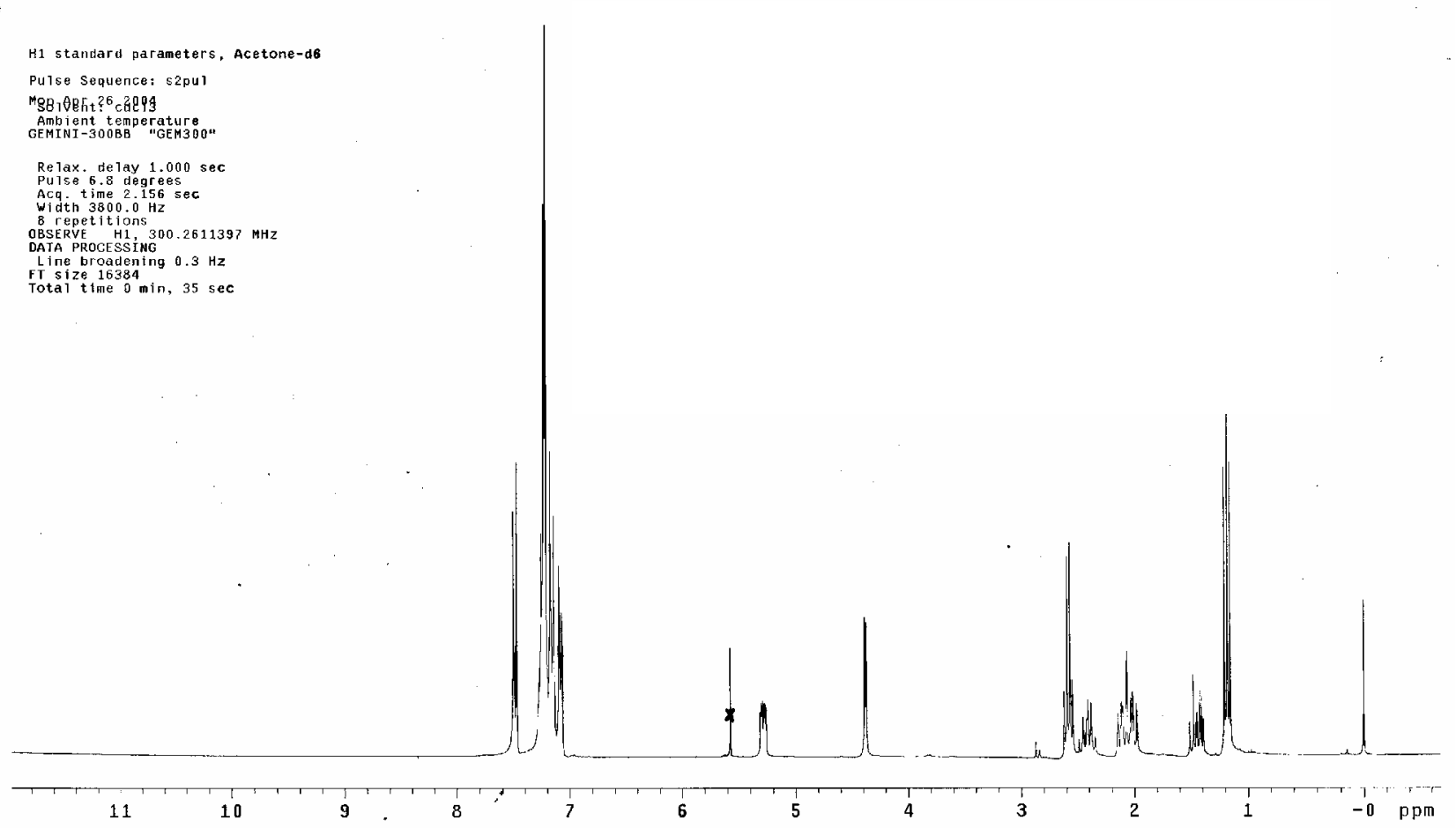

Page S32 


\section{Compound 5b}<smiles>CCc1ccc(N2C(=O)CCC2C(c2ccccc2)c2ccccc2)cc1</smiles>

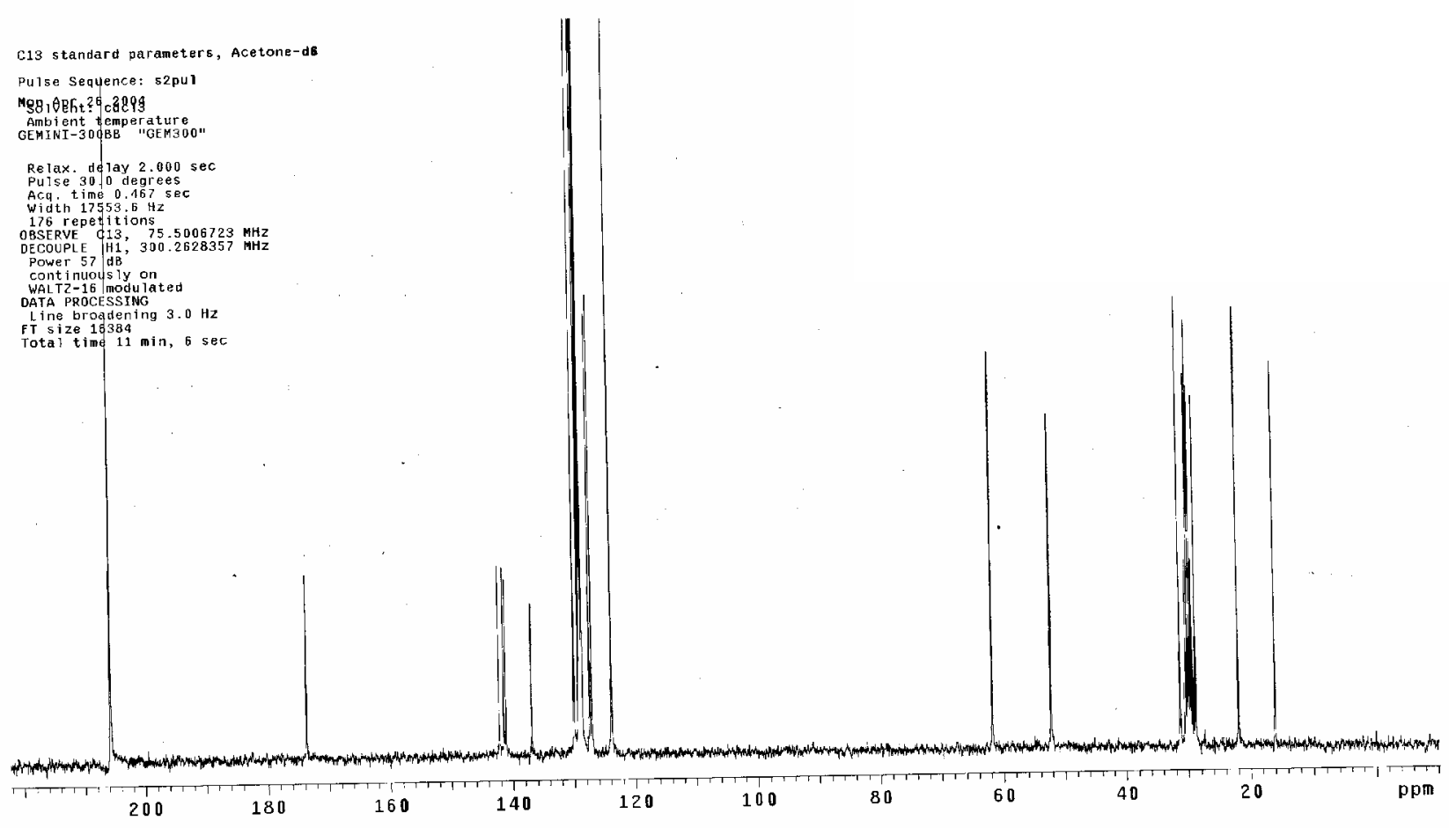


Compound 5c
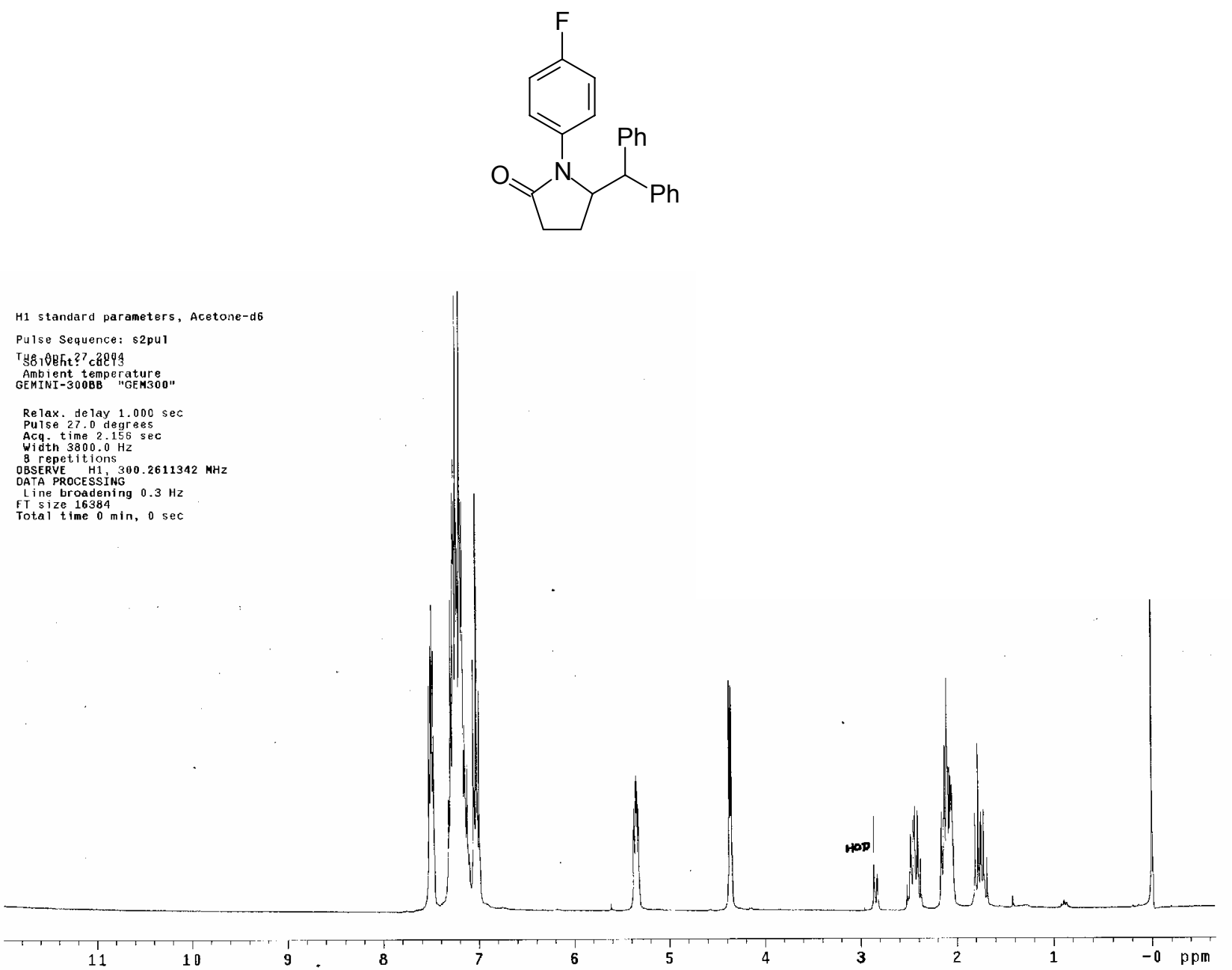
Compound $\mathbf{5 c}$
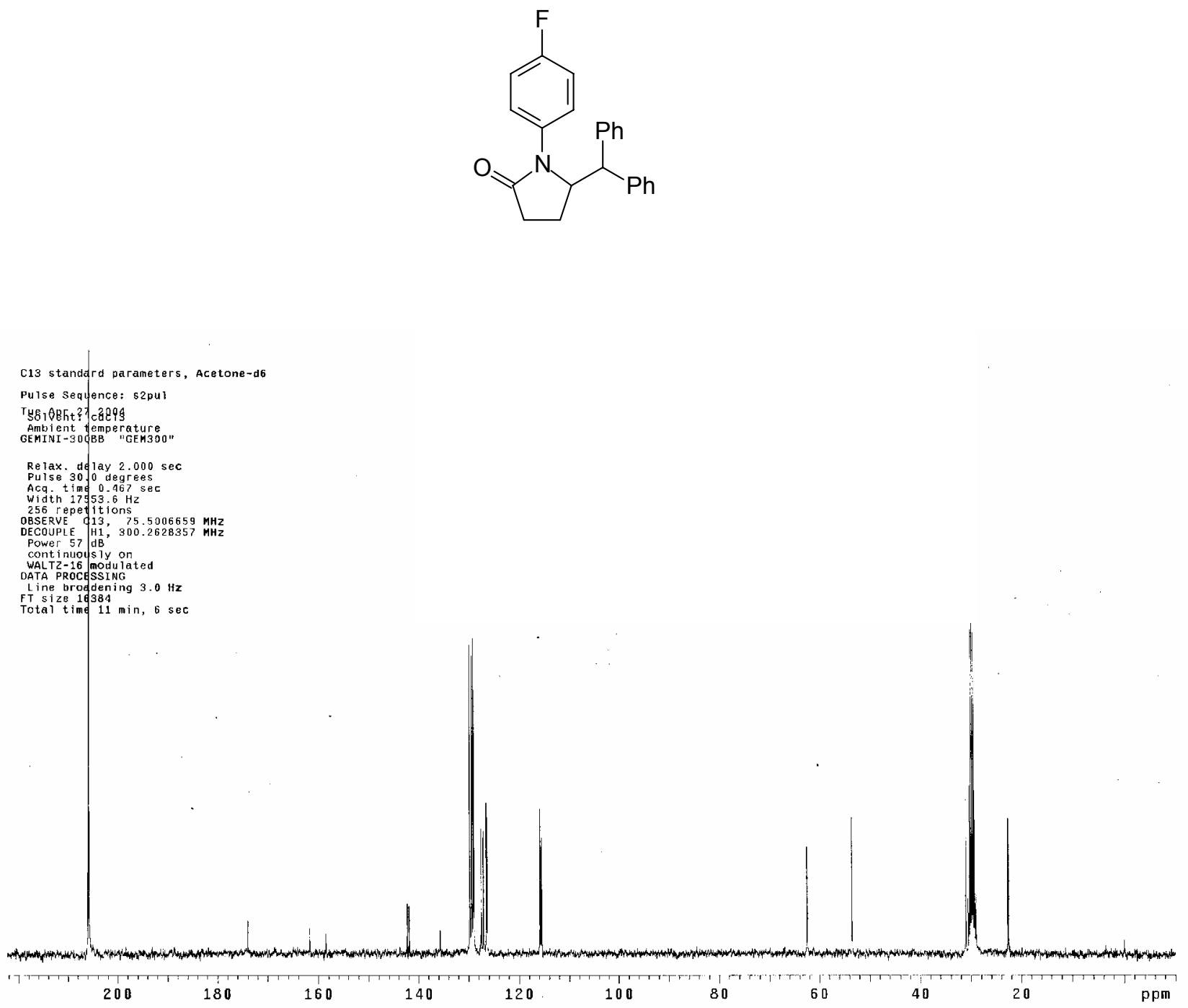

Page S35 


\section{Compound 5d}
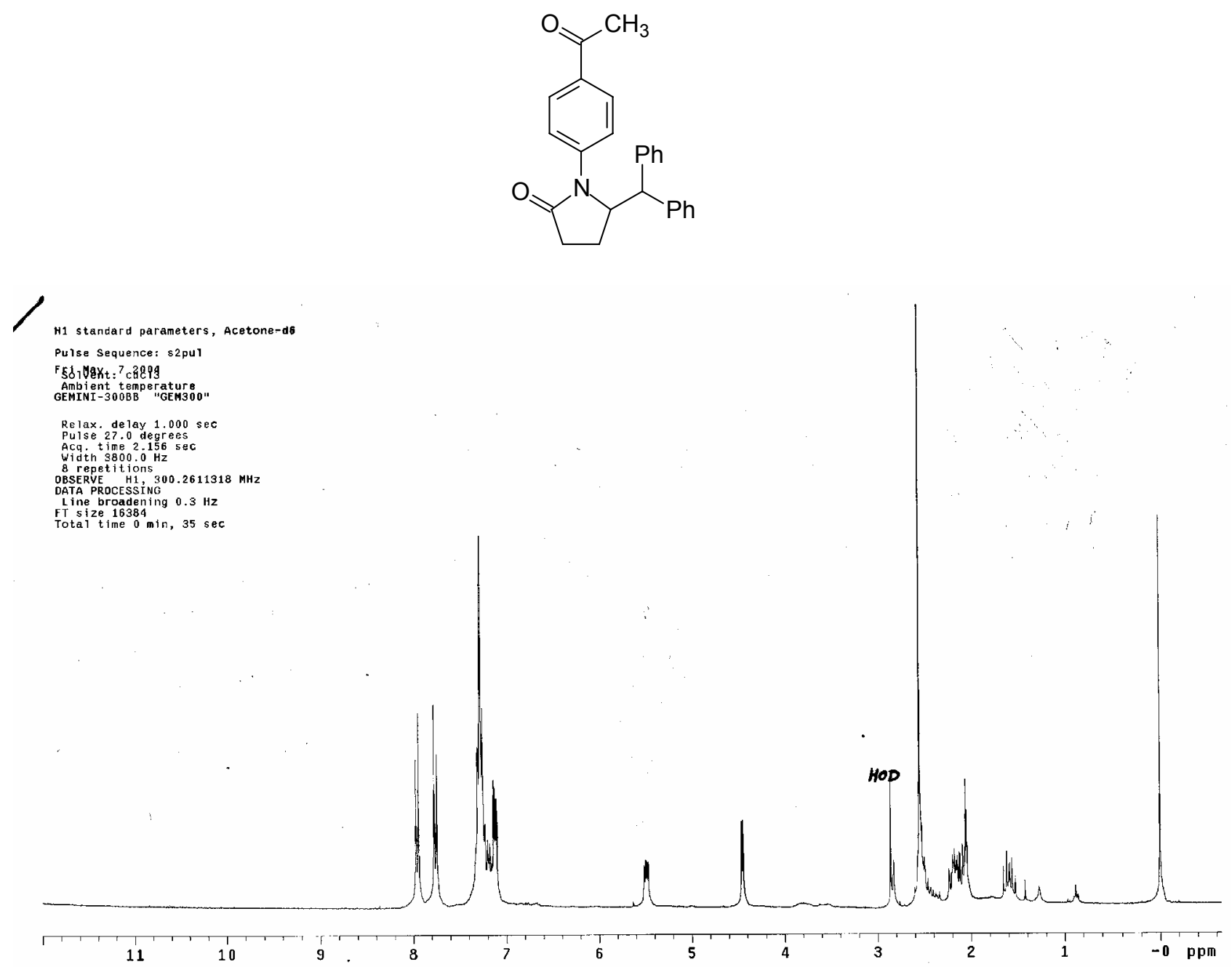


\section{Compound 5d}
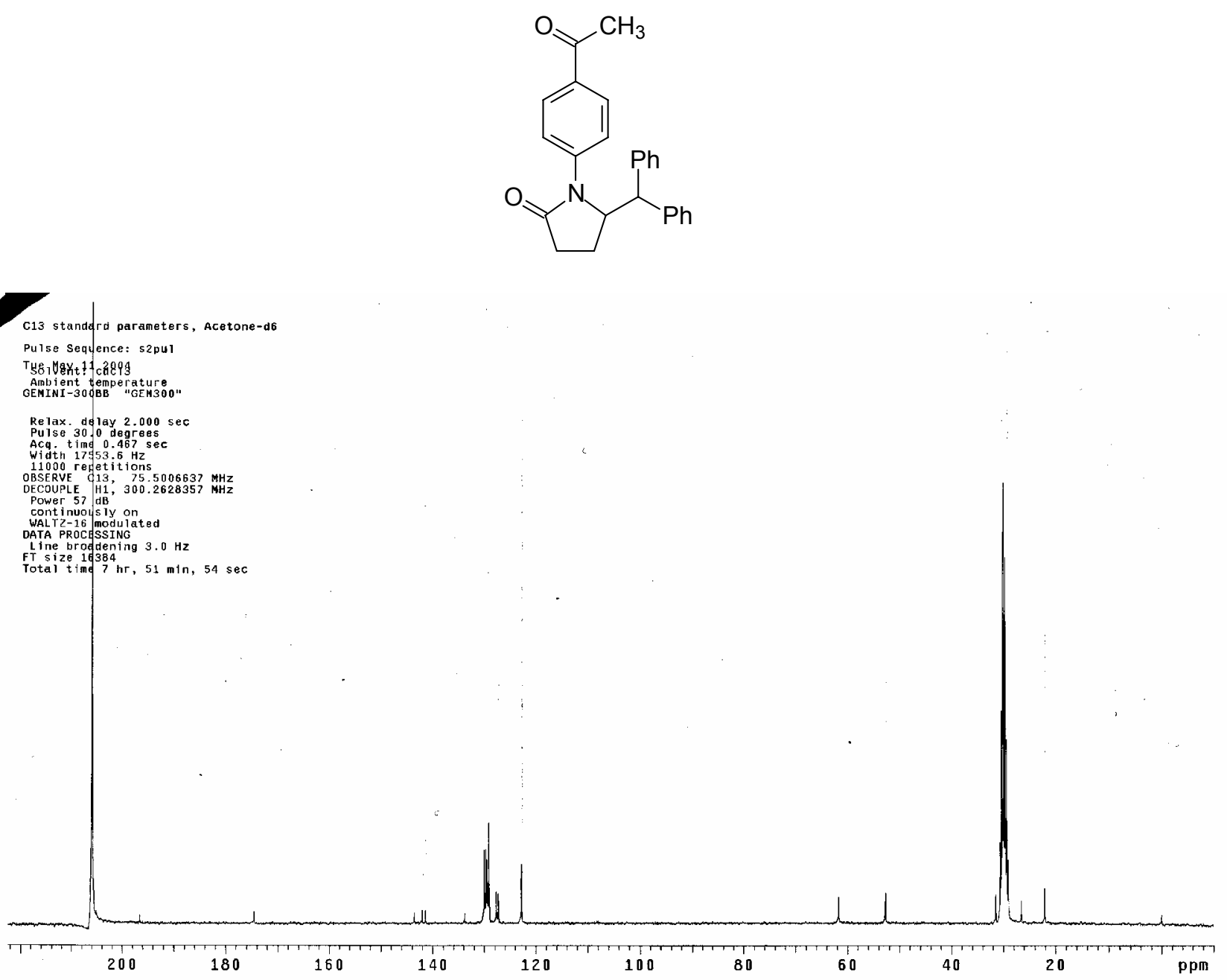
Compound 5e
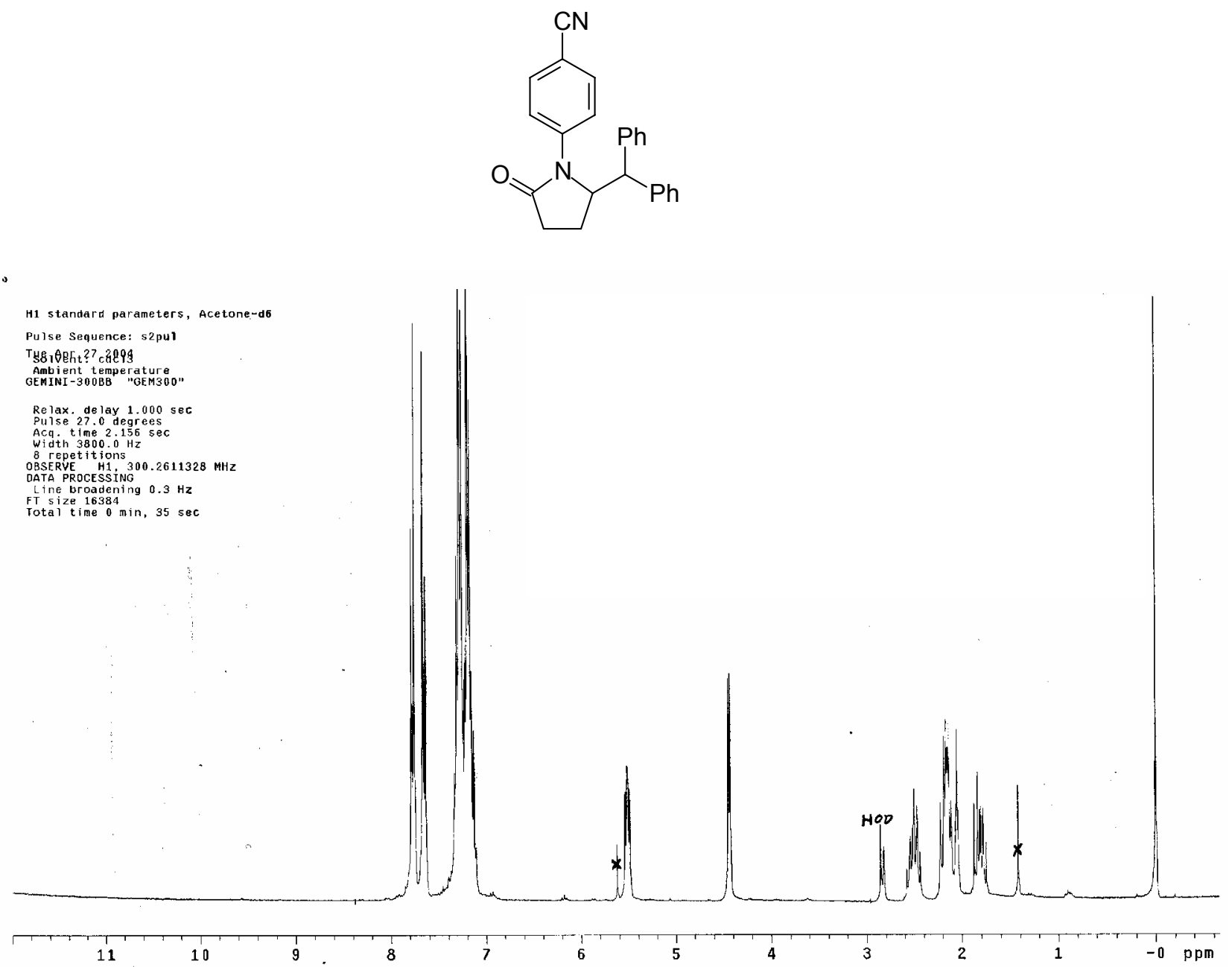

Page S38 


\section{Compound 5e}<smiles>N#Cc1ccc(N2C(=O)CCC2C(c2ccccc2)c2ccccc2)cc1</smiles>

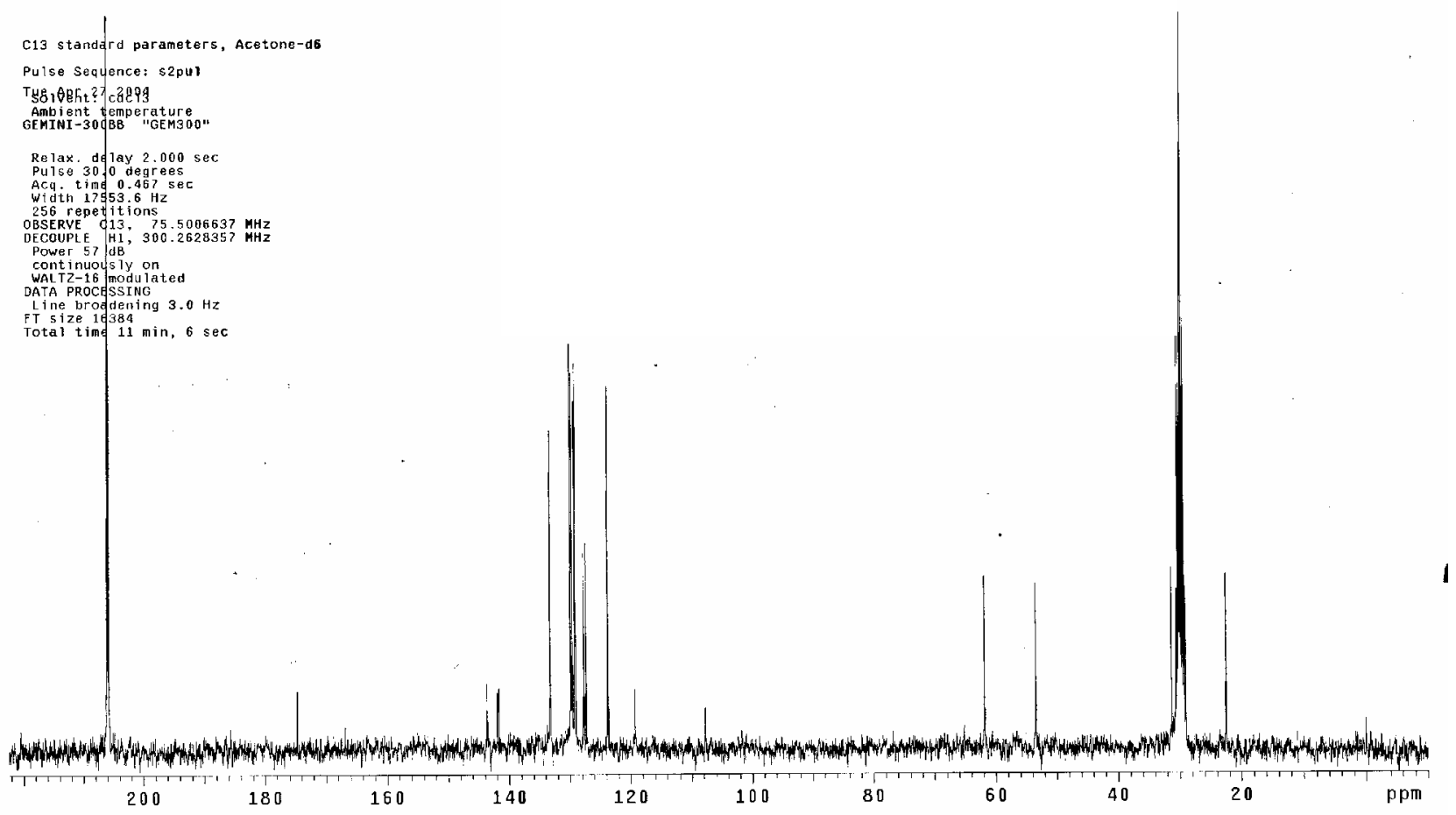




\section{Compound $\mathbf{5 f}$}

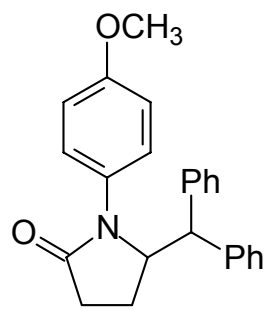

H1 standard parameters, Acetone-dis

Pulse Sequence: s2pul

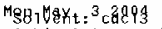

AEMIMI-300BB "GEM300"

Relax. de lay $1.000 \mathrm{sec}$

Pulse 3.6 degrees
Acq. time 2.156 sec

Wrepetitions

OBSERVE H1, 300.2611467 MHL

Line broadening $0.3 \mathrm{~Hz}$

FT size 16384 $0.3 \mathrm{~Hz}$

$0 \mathrm{~min}, 35 \mathrm{sec}$
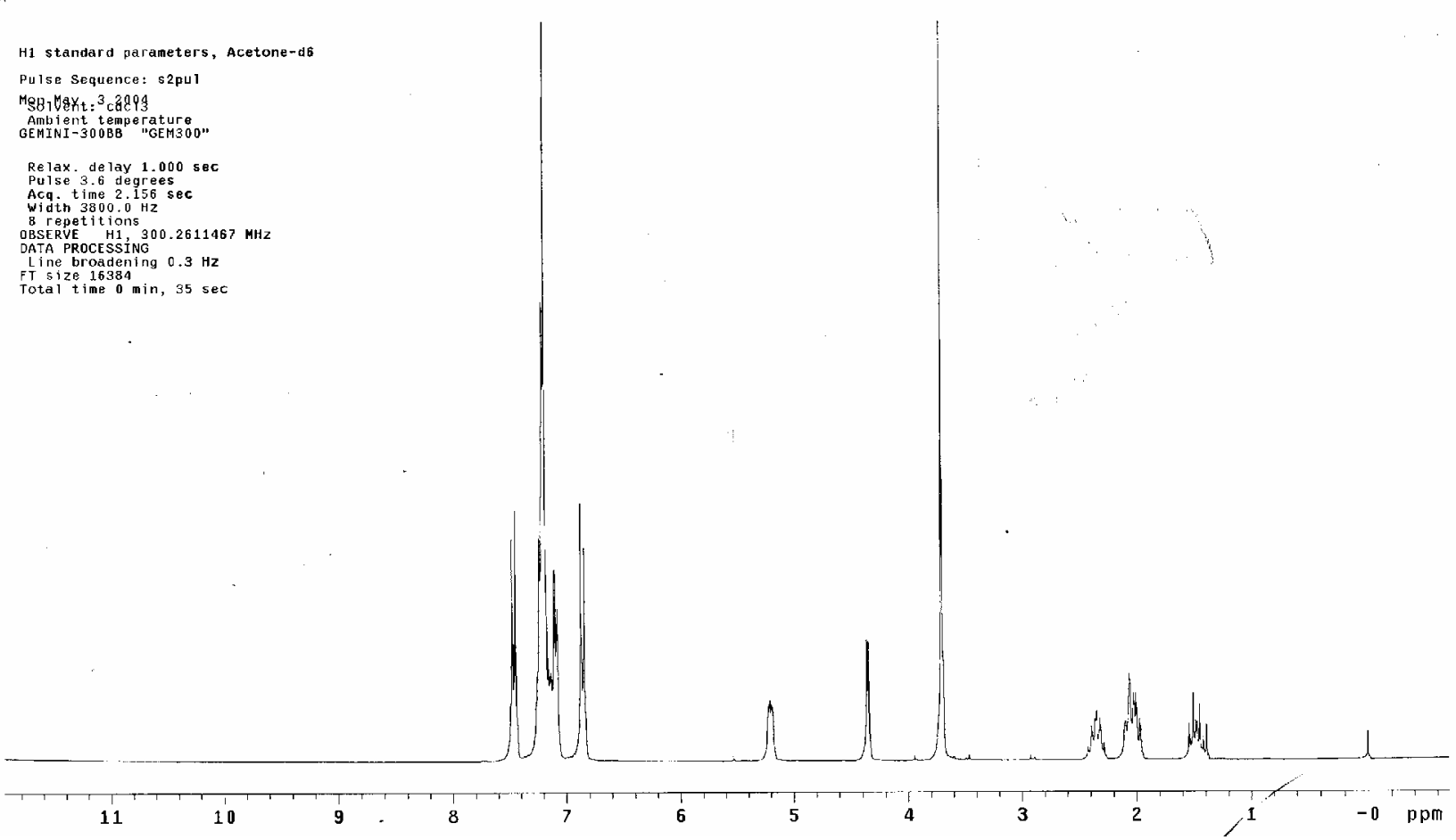

Page S40 


\section{Compound $\mathbf{5 f}$}
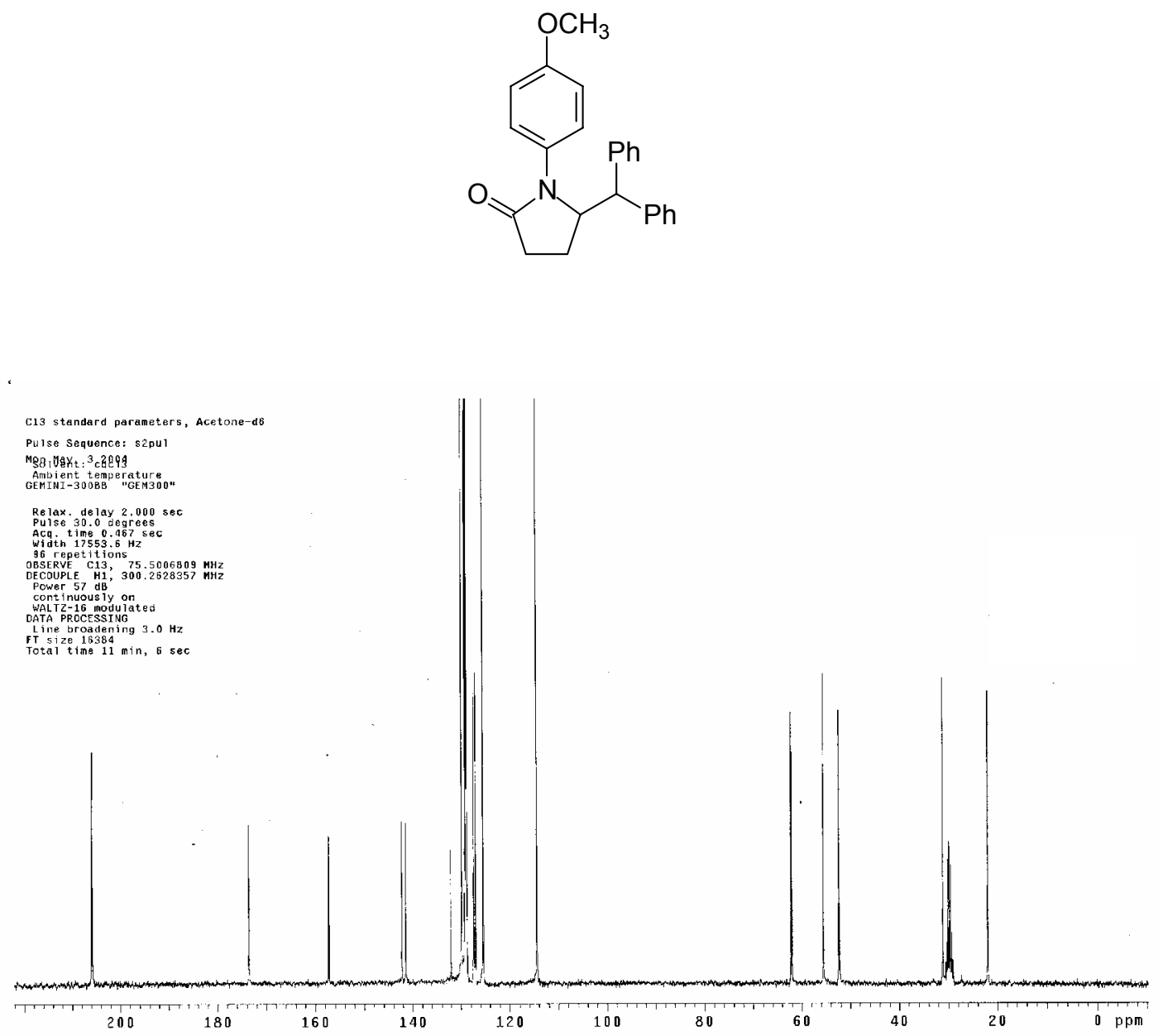

Page S41 


\section{$N$-(4-Methoxyphenyl)-6,6-diphenyl-5-hexenamide}<smiles>COc1ccc(NC(=O)CCCC=C(c2ccccc2)c2ccccc2)cc1</smiles>

1

H1 standard parameters, $\operatorname{CDC} 13$

Pulse Sequence: s2pul

Solvent: $\mathrm{CDC}_{13}$

Ambient temperature
Mercury-300BB "varian"

PULSE SEQUENCE

Relax. delay $2.000 \mathrm{sec}$

Pulse 49.1 degrees

Acq. $t$ ime 2.105 se

8 repetitions

OBSERVE H1, $299.8836749 \mathrm{MHZ}$

DATA PROCESSING

Line broadening $0.2 \mathrm{~Hz}$

Total time $0 \mathrm{~min}, 44 \mathrm{sec}$

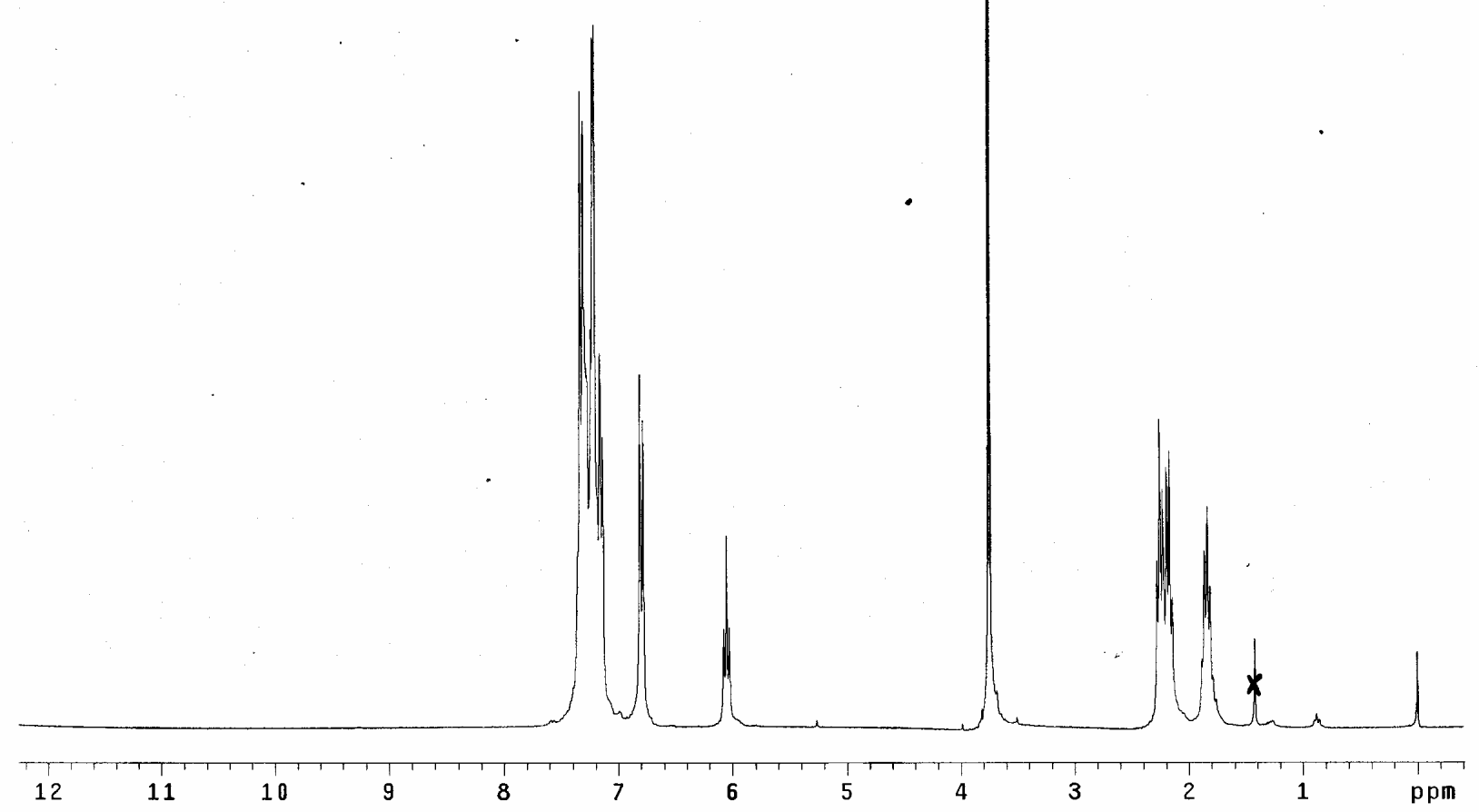




\section{$N$-(4-Methoxyphenyl)-6,6-diphenyl-5-hexenamide}<smiles>COc1ccc(NC(=O)CCCC=C(c2ccccc2)c2ccccc2)cc1</smiles>
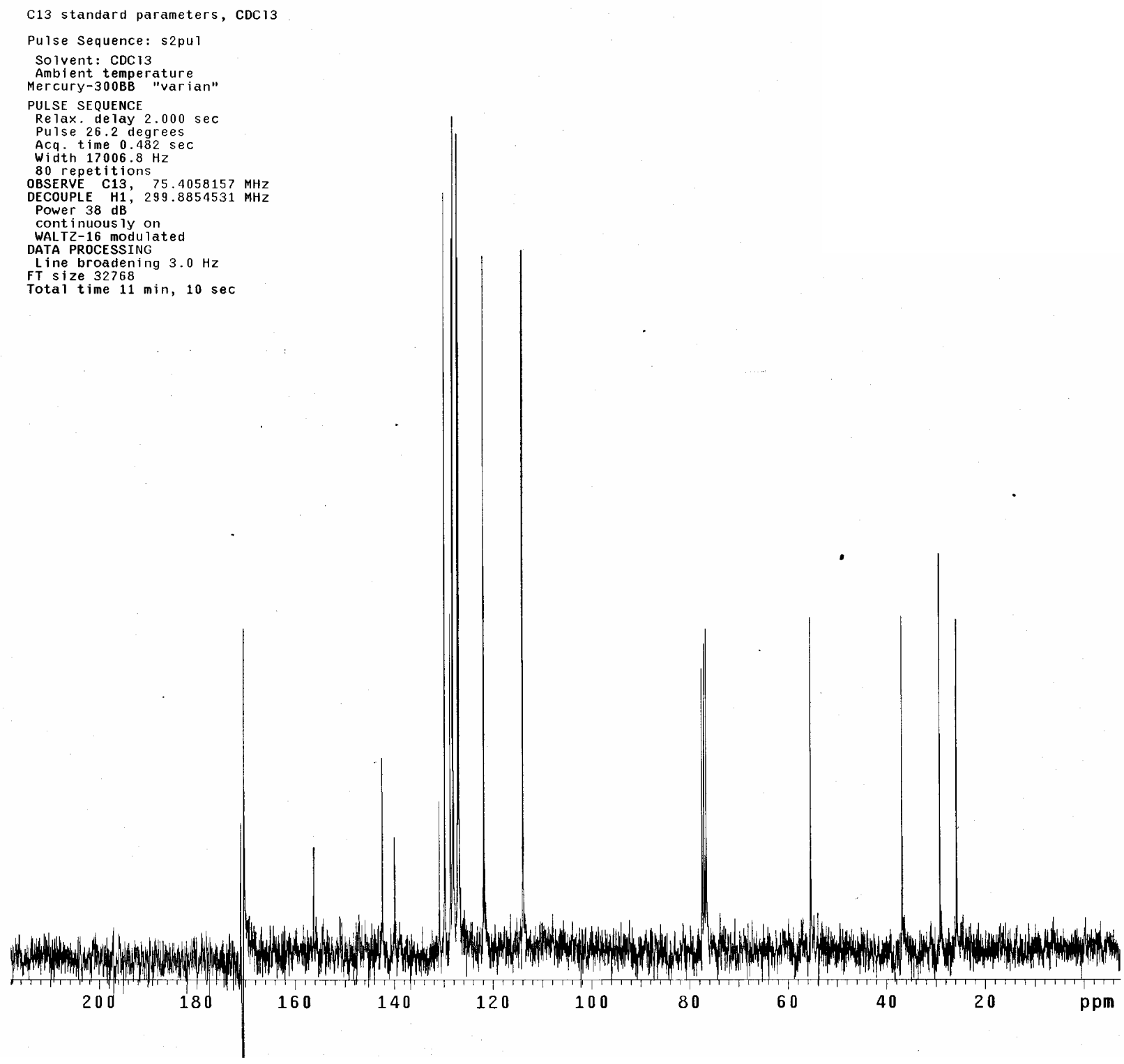


\section{$N$-(4-Methoxyphenyl)- $N$-(thiophenyl)-6,6-diphenyl-5-hexenamide}

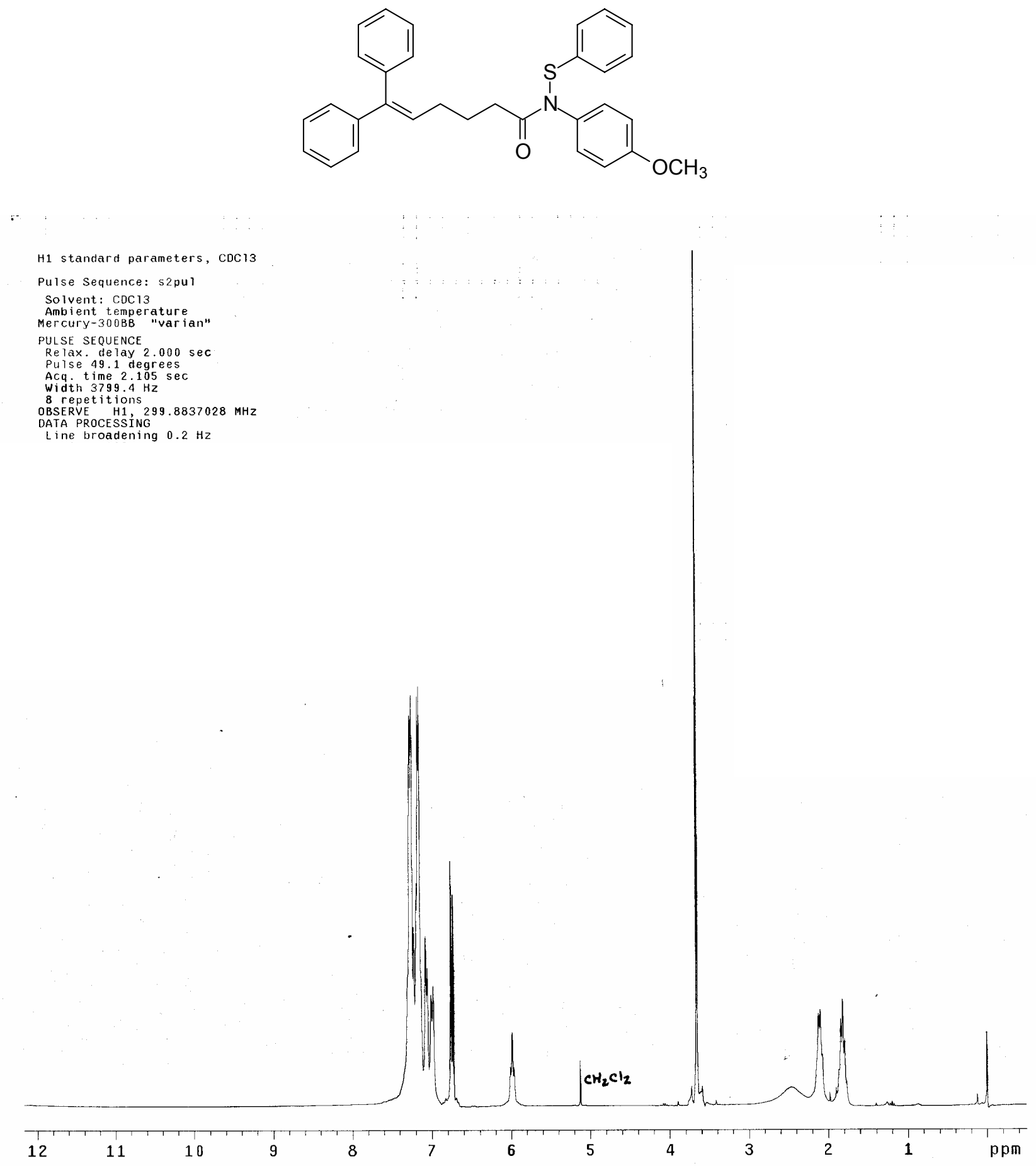




\section{$N$-(4-Methoxyphenyl)- $N$-(thiophenyl)-6,6-diphenyl-5-hexenamide}<smiles>COc1ccc(N(Sc2ccccc2)C(=O)CCCC=C(c2ccccc2)c2ccccc2)cc1</smiles>

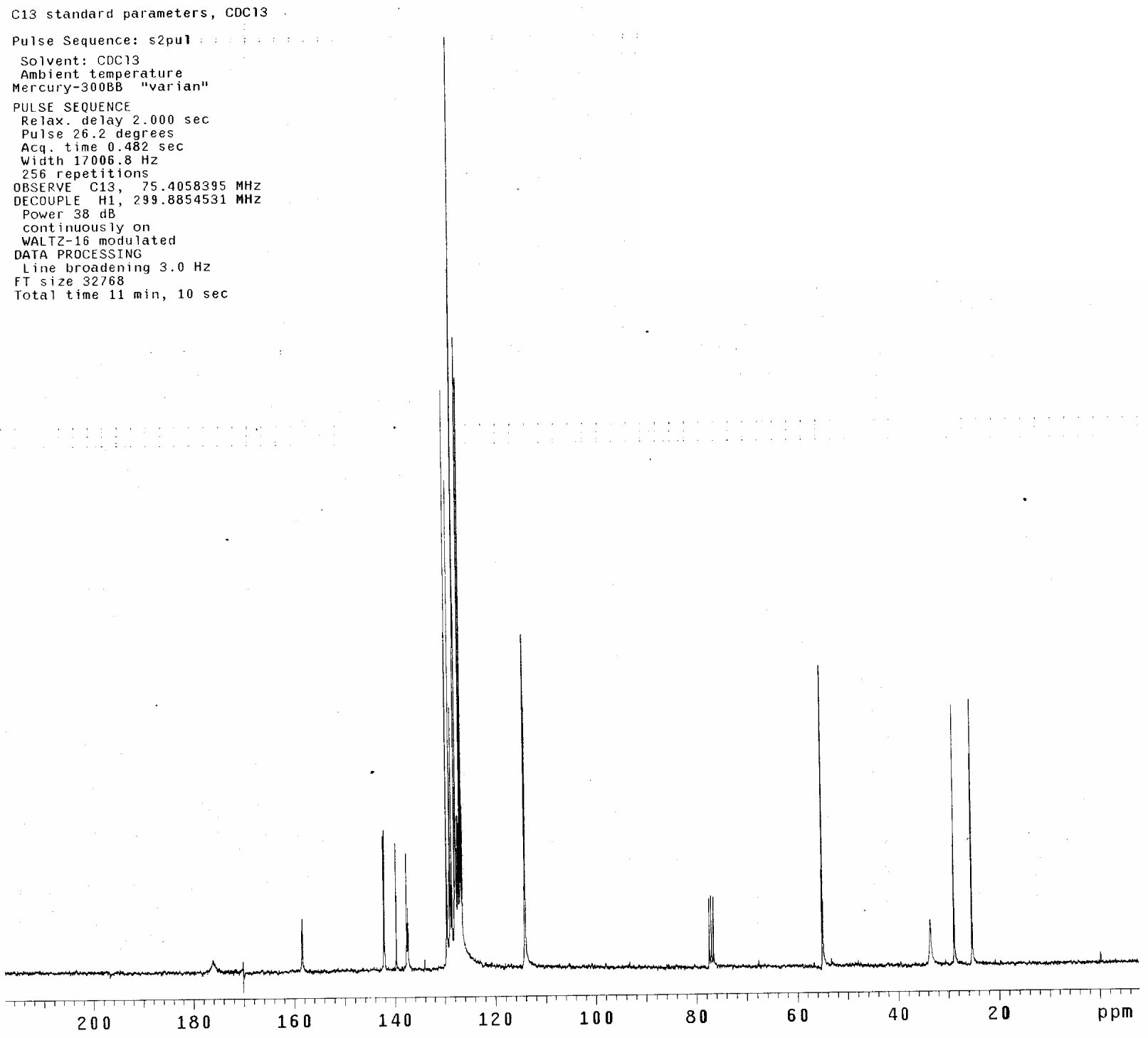

